\title{
Schrödinger type operators on generalized Morrey spaces
}

\author{
Pengtao $\mathrm{Li}^{1 *}$, Xin Wan² and Chuangyuan Zhang ${ }^{2}$
}

\footnotetext{
"Correspondence: ptli@qdu.edu.cn ${ }^{1}$ College of Mathematics, Qingdao University, Qingdao, Shandong 266071, China

Full list of author information is available at the end of the article
}

\begin{abstract}
In this paper we introduce a class of generalized Morrey spaces associated with the Schrödinger operator $L=-\Delta+V$. Via a pointwise estimate, we obtain the boundedness of the operators $V^{\beta_{2}}(-\Delta+V)^{-\beta_{1}}$ and their dual operators on these Morrey spaces.
\end{abstract}

MSC: Primary 42B35; 42B20

Keywords: generalized Morrey spaces; Schrödinger operator; commutator; reverse Hölder class

\section{Introduction}

The investigation of Schrödinger operators on the Euclidean space $\mathbb{R}^{n}$ with nonnegative potentials which belong to the reverse Hölder class has attracted attention of many authors. Shen [1] studied the Schrödinger operator $L=-\Delta+V$, assuming the nonnegative potential $V$ belongs to the reverse Hölder class $B_{q}, q \geq \frac{n}{2}$. In [1], Shen proved the $L^{p}$-boundedness of the operators $(-\Delta+V)^{i \gamma}, \nabla^{2}(-\Delta+V)^{-1}, \nabla(-\Delta+V)^{-1 / 2}$ and $\nabla(-\Delta+V)^{-1} \nabla$. For further information, we refer the reader to Guo et al. [2], Liu [3], Liu et al. [4, 5], Tang and Dong [6], Yang et al. [7, 8] and the references therein.

The purpose of this paper is to generalize the results of Shen [1] and Sugano [9] to a class of Morrey spaces associated with $L$, denoted by $L_{\alpha, \theta, V}^{p, q, \lambda}\left(\mathbb{R}^{n}\right)$. See Definition 2.8 below. The significance of these spaces is that for particular choices of the parameters $p, q, \lambda, \theta$ and $\alpha$, one obtains many classical function spaces (see Table 1 ).

In Section 3, let $T$ be one of the Schrödinger type operators $\nabla(-\Delta+V)^{-1} \nabla, \nabla(-\Delta+$ $V)^{-1 / 2}$ and $(-\Delta+V)^{-1 / 2} \nabla$. With the help of the $L^{p}$-boundedness of $T$, it is easy to verify that $T$ is bounded on $L_{\alpha, \theta, V}^{p, q, \lambda}\left(\mathbb{R}^{n}\right)$. For $b \in B M O\left(\mathbb{R}^{n}\right)$, we can also obtain the boundedness of the commutator $[b, T]$ on $L_{\alpha, \theta, V}^{p, q, \lambda}\left(\mathbb{R}^{n}\right)$. See Theorems 3.2 and 3.3. For $\theta=0, p=q$ and $0<\lambda<1, L_{\alpha, 0, V}^{p, p, \lambda}\left(\mathbb{R}^{n}\right)$ becomes the spaces $L_{\alpha, V}^{p, \lambda}\left(\mathbb{R}^{n}\right)$ introduced by Tang and Dong [6]. Hence, the results are generalizations of Theorems 1 and 2 in [6].

Table 1 Special cases of $L_{\alpha, \beta, v}^{p, q, \lambda}$

\begin{tabular}{ll}
\hline$\theta=0, \alpha=0, p=q, 0<\lambda<1$ & Morrey space $L^{p, \lambda}\left(\mathbb{R}^{n}\right)[10]$ \\
$\theta=0, p=q, 0<\lambda<1$ & Morrey type space $L_{\alpha, \lambda}^{p, \lambda}\left(\mathbb{R}^{n}\right)[6]$ \\
$\alpha=\lambda=0, \theta \in \mathbb{R}, 0<p, q<\infty$, & Herz spaces $K_{p}^{\theta, q}[11]$ \\
$\alpha=0, \lambda \geq 0, \theta \in \mathbb{R}, 0<p, q<\infty$ & Morrey-Herz spaces $M K_{p, q}^{\theta, \lambda}[12,13]$ \\
\hline
\end{tabular}

( $2015 \mathrm{Li}$ et al. This article is distributed under the terms of the Creative Commons Attribution 4.0 International License (http://creativecommons.org/licenses/by/4.0/), which permits unrestricted use, distribution, and reproduction in any medium, provided you give appropriate credit to the original author(s) and the source, provide a link to the Creative Commons license, and indicate if changes were made. 
In recent years, the fractional integral operator $I_{\alpha}=(-\Delta+V)^{-\alpha}$ has been studied extensively. We refer to Duong and Yan [14], Jiang [15], Tang and Dong [6] and Yang et al. [7] for details. Suppose that $V \in B_{s}, s \geq \frac{n}{2}$. For $0 \leq \beta_{2} \leq \beta_{1}<\frac{n}{2}$, let

$$
\left\{\begin{array}{l}
T_{\beta_{1}, \beta_{2}}=: V^{\beta_{2}}(-\Delta+V)^{-\beta_{1}}, \\
T_{\beta_{1}, \beta_{2}}^{*}=:(-\Delta+V)^{-\beta_{1}} V^{\beta_{2}} .
\end{array}\right.
$$

Sugano [9] obtained the weighted estimates for $T_{\beta_{1}, \beta_{2}}, T_{\beta_{1}, \beta_{2}}^{*}, 0<\beta_{2} \leq \beta_{1}<1$. If $\beta_{2}=0$, we can see that $T_{\beta_{1}, 0}=I_{\beta_{1}}$. So $T_{\beta_{1}, \beta_{2}}$ and $T_{\beta_{1}, \beta_{2}}^{*}$ can be seen as generalizations of $I_{\alpha}$. Moreover, for $\left(\beta_{1}, \beta_{2}\right)=(1,1)$ and $(1 / 2,1 / 2), T_{1,1}^{*}=(-\Delta+V)^{-1} V$ and $T_{1 / 2,1 / 2}^{*}=(-\Delta+V)^{-1 / 2} V^{1 / 2}$, respectively, which are studied by Shen [1] thoroughly. In Section 4 , assume that $1<p_{1}<\infty$, $1<p_{2}<s / \beta_{2}$ and $1<q<\infty$. If the index $\left(q, \beta_{1}, \beta_{2}, \lambda, \alpha, \theta\right)$ satisfies

$$
\left\{\begin{array}{l}
1 / p_{2}=1 / p_{1}-2\left(\beta_{1}-\beta_{2}\right) / n, \\
\alpha \in(-\infty, 0] \quad \text { and } \quad \lambda \in(0, n), \\
\lambda / q-1 / p_{1}+2 \beta_{1} / n<\theta<\lambda / q+1-1 / p_{1}
\end{array}\right.
$$

we prove that $T_{\beta_{1}, \beta_{2}}$ is bounded from $L_{\alpha, \theta, V}^{p_{1}, q, \lambda}\left(\mathbb{R}^{n}\right)$ to $L_{\alpha, \theta, V}^{p_{2}, q, \lambda}\left(\mathbb{R}^{n}\right)$. Specially, we know that $(-\Delta+V)^{-1} V$ and $(-\Delta+V)^{-1 / 2} V^{1 / 2}$ are bounded on $L_{\alpha, \theta, V}^{p, q, \lambda}\left(\mathbb{R}^{n}\right)$. See Theorems 4.7 and 4.8 for details.

In the research of harmonic analysis and partial differential equations, the commutators play an important role. If $T$ is a Calderón-Zygmund operator, $b \in B M O\left(\mathbb{R}^{n}\right)$, the $L^{p}$-boundedness of $[b, T]$ was first discovered by Coifman et al. [16]. Later, Strömberg [14] gave a simple proof, adopting the idea of relating commutators with the sharp maximal operator of Fefferman and Stein. In 2008, Guo et al. [2] introduced a condition $H(m)$ and obtained $L^{p}$-boundedness of the commutator of Riesz transforms associated with $L$, where $b \in B M O\left(\mathbb{R}^{n}\right)$. For further information, we refer to Liu [17], Liu et al. [4, 5], Yang et $a l$. [8] and the references therein.

In Section 5 , by the boundedness of $I_{\alpha}$ and $(-\Delta+V)^{-\beta} V^{\beta}$, we can deduce that the commutators $\left[b, T_{\beta_{1}, \beta_{2}}\right]$ and $\left[b, T_{\beta_{1}, \beta_{2}}^{*}\right]$ are bounded from $L^{p_{1}}\left(\mathbb{R}^{n}\right)$ to $L^{p_{2}}\left(\mathbb{R}^{n}\right)$ (see Theorem 5.1). Theorem 5.1 together with Lemmas 4.1 and 2.7 can be used to prove that the commutators $\left[b, T_{\beta_{1}, \beta_{2}}\right]$ and $\left[b, T_{\beta_{1}, \beta_{2}}^{*}\right]$ are bounded from $L_{\alpha, \theta, V}^{p_{1}, q, \lambda}\left(\mathbb{R}^{n}\right)$ to $L_{\alpha, \theta, V}^{p_{2}, q, \lambda}\left(\mathbb{R}^{n}\right)$, respectively (see Theorems 5.2 and 5.3).

Remark 1.1 Unlike the setting of the Lebesgue spaces, it is well known that the dual of $L^{p, \lambda}\left(\mathbb{R}^{n}\right)$ is not $L^{p^{\prime},-\lambda}\left(\mathbb{R}^{n}\right)$. Hence, after obtaining Theorem 4.7 , we cannot deduce Theorem 4.8 via the method of duality used by Guo et al. [2].

\section{Preliminaries}

\subsection{Schrödinger operator and the auxiliary function}

In this paper, we consider the Schrödinger differential operator $L=-\Delta+V$ on $\mathbb{R}^{n}, n \geq 3$, where $V$ is a nonnegative potential belonging to the reverse Hölder class $B_{s}, s \geq \frac{n}{2}$, which is defined as follows.

Definition 2.1 Let $V$ be a nonnegative function. 
(i) We say $V \in B_{s}, s>1$, if there exists $C>0$ such that for every ball $B \subset \mathbb{R}^{n}$, the reverse Hölder inequality

$$
\left(\frac{1}{|B|} \int_{B} V^{s}(x) d x\right)^{\frac{1}{s}} \lesssim\left(\frac{1}{|B|} \int_{B} V(x) d x\right)
$$

holds.

(ii) We say $V \in B_{\infty}$ if there exists a constant $C$ such that for every ball $B \subset \mathbb{R}^{n}$,

$$
\|V\|_{L^{\infty}(B)}=\frac{1}{|B|} \int_{B} V(x) d x .
$$

Remark 2.2 Assume $V \in B_{s}, 1<s<\infty$. Then $V(y) d y$ is a doubling measure. Namely, there exists a constant $C_{0}$ such that for any $r>0$ and $y \in \mathbb{R}^{n}$,

$$
\int_{B(x, 2 r)} V(y) d y \lesssim C_{0} \int_{B(x, r)} V(y) d y
$$

Definition 2.3 (Shen [1]) For $x \in \mathbb{R}^{n}$, the function $m_{V}(x)$ is defined as

$$
\frac{1}{m_{V}(x)}=: \sup \left\{r>0: \frac{1}{r^{n-2}} \int_{B(x, r)} V(y) d y \leq 1\right\} .
$$

Remark 2.4 The function $m_{V}$ reflects the scale of $V$ essentially, but behaves better. It is deeply studied in Shen [1] and plays a crucial role in our proof. We list a property of $m_{V}$ which will be used in the sequel and refer the reader to Guo et al. [2] for the details.

We state some notations and properties of $m_{V}$.

Lemma 2.5 (Lemma 1.4 in [1]) Suppose that $V \in B_{s}$ with $s \geq \frac{n}{2}$. Then there exist positive constants $C$ and $k_{0}$ such that

(a) if $|x-y| \leq \frac{C}{m_{V}(x)}, m_{V}(x) \sim m_{V}(y)$;

(b) $m_{V}(y) \lesssim\left(1+|x-y| m_{V}(x)\right)^{k_{0}} m_{V}(x)$;

(c) $m_{V}(y) \geq C m_{V}(x) /\left\{1+|x-y| m_{V}(x)\right\}^{k_{0} /\left(k_{0}+1\right)}$.

Lemma 2.6 (Lemma 1.2 in [1]) Suppose that $V \in B_{s}, s>\frac{n}{2}$. There exists a constant $C$ such that for $0<r<R<\infty$,

$$
\frac{1}{r^{n-2}} \int_{B(x, r)} V(y) d y \lesssim\left(\frac{R}{r}\right)^{\frac{n}{s}-2} \cdot \frac{1}{R^{n-2}} \int_{B(x, R)} V(y) d y .
$$

Lemma 2.7 (Lemma 2.3 in [2]) Suppose $V \in B_{s}, s>\frac{n}{2}$. Then, for any $N>\log _{2} C_{0}+1$, there exists a constant $C_{N}$ such that for any $x \in \mathbb{R}^{n}$ and $r>0$,

$$
\frac{1}{\left(1+r m_{V}(x)\right)^{N}} \int_{B(x, r)} V(y) d y \lesssim C_{N} r^{n-2} .
$$

\subsection{Generalized Morrey spaces associated with $L$}

Suppose that $V \in B_{s}, s>1$. Let $L=-\Delta+V$ be the Schrödinger operator. Now we introduce a class of generalized Morrey spaces associated with $L$. For $k \in \mathbb{Z}$, let $E_{k}=$ $B\left(x_{0}, 2^{k} r\right) \backslash B\left(x_{0}, 2^{k-1} r\right)$ and $\chi_{k}$ be the characteristic function of $E_{k}$. 
Definition 2.8 Suppose that $V \in B_{s}, s>1$. Let $p \in[1,+\infty), q \in[1,+\infty), \alpha \in(-\infty,+\infty)$ and $\lambda \in(0, n), \theta \in(-\infty,+\infty)$. For $f \in L_{\mathrm{loc}}^{q}\left(\mathbb{R}^{n}\right)$, we say $f \in L_{\alpha, \theta, V}^{p, q, \lambda}\left(\mathbb{R}^{n}\right)$ provided that

$$
\|f\|_{L_{\alpha, \theta, V}^{p, q, \lambda}\left(\mathbb{R}^{n)}\right.}^{q}=\sup _{B\left(x_{0}, r\right) \subset \mathbb{R}^{n}} \frac{\left(1+r m_{V}\left(x_{0}\right)\right)^{\alpha}}{r^{\lambda n}} \sum_{k=-\infty}^{0}\left|E_{k}\right|^{\theta q}\left\|\chi_{k} f\right\|_{L^{p}\left(\mathbb{R}^{n}\right)}^{q}<\infty
$$

where $B\left(x_{0}, r\right)$ denotes a ball centered at $x_{0}$ and with radius $r$.

\section{Proposition 2.9}

(i) For $\alpha_{1}>\alpha_{2}, L_{\alpha_{1}, \theta, V}^{p, q, \lambda}\left(\mathbb{R}^{n}\right) \subseteq L_{\alpha_{2}, \theta, V}^{p, \lambda, q}\left(\mathbb{R}^{n}\right)$.

(ii) If $\theta=0, p=q$ and $\alpha<0, L^{p, \lambda}\left(\mathbb{R}^{n}\right) \subset L_{\alpha, \theta, V}^{p, q, \lambda}\left(\mathbb{R}^{n}\right)$.

(iii) If $\theta=0, p=q$ and $\alpha>0, L_{\alpha, \theta, V}^{p, q, \lambda}\left(\mathbb{R}^{n}\right) \subset L^{p, \lambda}\left(\mathbb{R}^{n}\right)$.

\subsection{Calderón-Zygmund operators}

We say that an operator $T$ taking $C_{c}^{\infty}\left(\mathbb{R}^{n}\right)$ into $L_{\text {loc }}^{1}\left(\mathbb{R}^{n}\right)$ is called a Calderón-Zygmund operator if

(a) $T$ extends to a bounded linear operator on $L^{2}\left(\mathbb{R}^{n}\right)$;

(b) there exists a kernel $K$ such that for every $f \in L_{\text {loc }}^{1}\left(\mathbb{R}^{n}\right)$,

$$
T f(x)=\int_{\mathbb{R}^{n}} K(x, y) f(y) d y \quad \text { a.e. on }\{\operatorname{supp} f\}^{c} ;
$$

(c) the kernel $K(x, y)$ satisfies the Calderón-Zygmund estimate

$$
\begin{aligned}
& |K(x, y)| \leq \frac{C}{|x-y|^{n}} ; \\
& |K(x+h, y)-K(x, y)|+|K(x, y+h)-K(x, y)| \leq \frac{C|h|^{\delta}}{|x-y|^{n+\delta}}
\end{aligned}
$$

for $x, y \in \mathbb{R}^{n},|h|<\frac{|x-y|}{2}$ and for some $\delta>0$.

Shen [1] obtained the following result.

Theorem 2.10 (Theorem 0.8 in [1]) Suppose $V \in B_{n}$. Then

$$
\nabla(-\Delta+V)^{-1} \nabla, \quad \nabla(-\Delta+V)^{-\frac{1}{2}} \text { and }(-\Delta+V)^{-\frac{1}{2}} \nabla
$$

are Calderón-Zygmund operators.

Corollary 2.11 Suppose that $V \in B_{n}$ and $b \in B M O\left(\mathbb{R}^{n}\right)$. The commutator $[b, T]$ is bounded on $L^{p}\left(\mathbb{R}^{n}\right)$.

In particular, let $K$ denote the kernel of one of the above operators. Then $K$ satisfies the following estimate:

$$
|K(x, y)| \leq \frac{C_{N}}{\left(1+|x-y| m_{V}(x)\right)^{N}} \frac{1}{|x-y|^{n}}
$$

for any $N \in \mathbb{N}$. See (6.5) of Shen [1] for details. 
Suppose $V \in B_{s}$ for $s \geq \frac{n}{2}$. Let $L=-\Delta+V$. The semigroup generated by $L$ is defined as

$$
T_{t} f(x)=e^{-t L} f(x)=\int_{\mathbb{R}^{n}} K_{t}(x, y) f(y) d y, \quad f \in L^{2}\left(\mathbb{R}^{n}\right), t>0,
$$

where $K_{t}$ is the kernel of $e^{-t L}$.

Lemma 2.12 ([18]) Let $K_{t}(x, y)$ be as in (2.3). For every nonnegative integer $k$, there is a constant $C_{k}$ such that

$$
0 \leq K_{t}(x, y) \leq C_{k} t^{-\frac{n}{2}} \exp \left(-|x-y|^{2} / 5 t\right)\left(1+\sqrt{t} m_{V}(x)+\sqrt{t} m_{V}(y)\right)^{-k} .
$$

Some notations Throughout the paper, $c$ and $C$ will denote unspecified positive constants, possibly different at each occurrence. The constants are independent of the functions. $\mathrm{U} \approx \mathrm{V}$ represents that there is a constant $c>0$ such that $c^{-1} \mathrm{~V} \leq \mathrm{U} \leq c \mathrm{~V}$ whose right inequality is also written as $\mathrm{U} \lesssim \mathrm{V}$. Similarly, if $\mathrm{V} \geq c \mathrm{U}$, we denote $\mathrm{V} \gtrsim \mathrm{U}$.

\section{Riesz transforms and the commutators on $L_{\alpha, \theta, v}^{p, q, \lambda}\left(\mathbb{R}^{n}\right)$}

Throughout this paper, for $p \in(1, \infty)$, denote by $p^{\prime}$ the conjugate of $p$, that is, $\frac{1}{p}+\frac{1}{p^{\prime}}=1$. Let $V \in B_{n}$. In this section, we assume that $T$ is one of the Schrödinger type operators $\nabla(-\Delta+V)^{-1} \nabla, \nabla(-\Delta+V)^{-1 / 2}$ and $(-\Delta+V)^{-1 / 2} \nabla$. We study the boundedness on $L_{\alpha, \theta, V}^{p, q, \lambda}\left(\mathbb{R}^{n}\right)$ of $T$ and its commutator $[b, T]$ with $b \in B M O\left(\mathbb{R}^{n}\right)$. The bounded mean oscillation space $B M O\left(\mathbb{R}^{n}\right)$ is defined as follows.

Definition 3.1 A locally integrable function $b$ is said to belong to $B M O\left(\mathbb{R}^{n}\right)$ if

$$
\|b\|_{B M O}=: \sup _{B} \frac{1}{|B|} \int_{B}\left|b(x)-b_{B}\right| d x<\infty
$$

where the supremum is taken over all balls $B$ in $\mathbb{R}^{n}$. Here $b_{B}=\frac{1}{|B|} \int_{B} b(x) d x$ stands for the mean value of $b$ over the ball $B$ and $|B|$ means the measure of $B$.

We first prove that $T$ is bounded on $L_{\alpha, \theta, V}^{p, q, \lambda}\left(\mathbb{R}^{n}\right)$.

Theorem 3.2 Suppose that $\alpha \in(-\infty, 0], \lambda \in(0, n)$ and $1<q<\infty$. If $1<p<\infty, \frac{\lambda}{q}-\frac{1}{p}<\theta<$ $\frac{\lambda}{q}+1-\frac{1}{p}$, then the operators $T$ are bounded on $L_{\alpha, \theta, V}^{p, q, \lambda}\left(\mathbb{R}^{n}\right)$.

Proof For any ball $B\left(x_{0}, r\right)$, write

$$
f(y)=\sum_{j=-\infty}^{\infty} f(y) \chi_{j}(y)=\sum_{j=-\infty}^{\infty} f_{j}(y),
$$

where $E_{j}=B\left(x_{0}, 2^{j} r\right) \backslash B\left(x_{0}, 2^{j-1} r\right)$. Hence, we have

$$
\begin{aligned}
& \left(1+r m_{V}\left(x_{0}\right)\right)^{\alpha} r^{\lambda n} \sum_{k=-\infty}^{0}\left|E_{k}\right|^{\theta q}\left\|\chi_{k} T f\right\|_{L^{p}\left(\mathbb{R}^{n)}\right.}^{q} \\
& \lesssim\left(1+r m_{V}\left(x_{0}\right)\right)^{\alpha} r^{-\lambda n} \sum_{k=-\infty}^{0}\left|E_{k}\right|^{\theta q}\left(\sum_{j=-\infty}^{k-2}\left\|\chi_{k} T f_{j}\right\|_{L^{p}\left(\mathbb{R}^{n}\right)}\right)^{q}
\end{aligned}
$$




$$
\begin{aligned}
& +\left(1+r m_{V}\left(x_{0}\right)\right)^{\alpha} r^{-\lambda n} \sum_{k=-\infty}^{0}\left|E_{k}\right|^{\theta q}\left(\sum_{j=k-1}^{k+1}\left\|\chi_{k} T f_{j}\right\|_{L^{p}\left(\mathbb{R}^{n}\right)}\right)^{q} \\
& +\left(1+r m_{V}\left(x_{0}\right)\right)^{\alpha} r^{-\lambda n} \sum_{k=-\infty}^{0}\left|E_{k}\right|^{\theta q}\left(\sum_{j=k+2}^{\infty}\left\|\chi_{k} T f_{j}\right\|_{L^{p}\left(\mathbb{R}^{n}\right)}\right)^{q} \\
& =A_{1}+A_{2}+A_{3} .
\end{aligned}
$$

For $A_{2}$, by Theorem 2.10 , we have

$$
\begin{aligned}
A_{2} & \lesssim\left(1+r m_{V}\left(x_{0}\right)\right)^{\alpha} r^{-\lambda n} \sum_{k=-\infty}^{0}\left|E_{k}\right|^{\theta q}\left(\sum_{j=k-1}^{k+1}\left\|T f_{j}\right\|_{L^{p}\left(\mathbb{R}^{n}\right)}\right)^{q} \\
& \lesssim\left(1+r m_{V}\left(x_{0}\right)\right)^{\alpha} r^{-\lambda n} \sum_{k=-\infty}^{0}\left|E_{k}\right|^{\theta q}\left(\sum_{j=k-1}^{k+1}\left\|f_{j}\right\|_{L^{p}\left(\mathbb{R}^{n)}\right)}\right)^{q} \\
& \lesssim\|f\|_{L_{\alpha, \theta, V}^{p, q, \lambda}}^{q} .
\end{aligned}
$$

We first estimate the term $E_{1}$. Note that if $x \in E_{k}, y \in E_{j}$ and $j \leq k-2$, then $|x-y| \sim 2^{k} r$. By Lemma 2.5 and (2.2), we can get

$$
\begin{aligned}
\left\|\chi_{k} T f_{j}\right\|_{L^{p}\left(\mathbb{R}^{n}\right)} & \lesssim\left(\int_{E_{k}}\left|\int_{\mathbb{R}^{n}} \frac{1}{\left(1+|x-y| m_{V}(x)\right)^{N}} \frac{1}{|x-y|^{n}}\right| f_{j}(y)|d y|^{p} d x\right)^{\frac{1}{p}} \\
& \lesssim \frac{1}{\left(1+2^{k} r m_{V}\left(x_{0}\right)\right)^{N / k_{0}+1}} \frac{1}{\left(2^{k} r\right)^{n}}\left|E_{k}\right|^{\frac{1}{p}} \int_{E_{j}}|f(y)| d y \\
& \lesssim \frac{1}{\left(1+2^{k} r m_{V}\left(x_{0}\right)\right)^{N / k_{0}+1}}\left|E_{k}\right|^{\frac{1}{p}-1}\left|E_{j}\right|^{\frac{1}{p^{p}}}\left(\int_{E_{j}}|f(y)|^{p} d y\right)^{\frac{1}{p}}
\end{aligned}
$$

where $\frac{1}{p}+\frac{1}{p^{\prime}}=1$. Since $-\frac{1}{p}+\frac{\lambda}{q}<\theta<\left(1-\frac{1}{p}\right)+\frac{\lambda}{q}$, we obtain

$$
\begin{aligned}
A_{1} \lesssim\left(1+r m_{V}\left(x_{0}\right)\right)^{\alpha} r^{-\lambda n} \sum_{k=-\infty}^{0}\left|E_{k}\right|^{\theta q}\left(\sum_{j=-\infty}^{k-2} \frac{\left|E_{k}\right|^{\frac{1}{p}-1}\left|E_{j}\right|^{\frac{1}{p^{\prime}}}\left\|\chi_{j} f\right\|_{L^{p}\left(\mathbb{R}^{n}\right)}}{\left(1+2^{k} r m_{V}\left(x_{0}\right)\right)^{N / k_{0}+1}}\right)^{q} \\
\lesssim\left(1+r m_{V}\left(x_{0}\right)\right)^{\alpha} r^{-\lambda n} \sum_{k=-\infty}^{0}\left|E_{k}\right|^{\theta q}\left(\sum_{j=-\infty}^{k-2} \frac{2^{\frac{n(j-k)}{p^{\prime}}}\left(1+2^{j} r m_{V}\left(x_{0}\right)\right)^{-\frac{\alpha}{q}}}{\left(1+2^{k} r m_{V}\left(x_{0}\right)\right)^{N / k_{0}+1}}\right. \\
\left.\quad \times\left(2^{j} r\right)^{\frac{\lambda n}{q}}\left|E_{j}\right|^{-\theta}\left(1+2^{j} r m_{V}\left(x_{0}\right)\right)^{\frac{\alpha}{q}}\left(2^{j} r\right)^{-\frac{\lambda n}{q}}\left(\left|E_{j}\right|^{\theta q}\left\|\chi_{j} f\right\|_{L^{p}\left(\mathbb{R}^{n}\right)}^{q}\right)^{\frac{1}{q}}\right)^{q} \\
\lesssim\left(1+r m_{V}\left(x_{0}\right)\right)^{\alpha} r^{-\lambda n} \sum_{k=-\infty}^{0}\left|E_{k}\right|^{\lambda}\left(\sum_{j=-\infty}^{k-2} 2^{\left.\frac{n(j-k)}{p^{\prime}}\left|E_{k}\right|^{\theta-\frac{\lambda}{q}}\left|E_{j}\right|^{\frac{\lambda}{q}-\theta}\right)^{q}\|f\|_{L_{\alpha, \theta, V}^{p, q, \lambda}\left(\mathbb{R}^{n}\right)}^{q}}\right. \\
\lesssim\left(1+r m_{V}\left(x_{0}\right)\right)^{\alpha} r^{-\lambda n} \sum_{k=-\infty}^{0}\left|E_{k}\right|^{\lambda}\left(\sum_{j=-\infty}^{k-2} 2^{(j-k) n\left(1-\frac{1}{p}+\frac{\lambda}{q}-\theta\right)}\right)^{q}\|f\|_{L_{\alpha, \theta, V}^{p, q, \lambda}\left(\mathbb{R}^{n}\right)}^{q} \\
\lesssim\|f\|_{L_{\alpha, \theta, V}^{p} q, \lambda\left(\mathbb{R}^{n}\right)}^{\cdot}
\end{aligned}
$$


For $A_{3}$, we can see that when $x \in E_{k}, y \in E_{j}$, then $|x-y| \sim 2^{j} r$ for $j \geq k+2$. Similar to $E_{1}$, we have

$$
\begin{aligned}
\left\|\chi_{k} T f_{j}\right\|_{L^{p}\left(\mathbb{R}^{n}\right)} & \lesssim \frac{1}{\left(1+2 j r m_{V}\left(x_{0}\right)\right)^{N / k_{0}+1}} \frac{1}{(2 j r)^{n}}\left|E_{k}\right|^{\frac{1}{p}} \int_{E_{j}}|f(y)| d y \\
& \lesssim \frac{1}{\left(1+2 j r m_{V}\left(x_{0}\right)\right)^{N / k_{0}+1}} \frac{1}{(2 j r)^{n}}\left|E_{k}\right|^{\frac{1}{p}}\left|E_{j}\right|^{\frac{1}{p^{\prime}}}\left(\int_{E_{j}}|f(y)|^{p} d y\right)^{\frac{1}{p}} \\
& \lesssim \frac{1}{\left(1+2 j r m_{V}\left(x_{0}\right)\right)^{N / k_{0}+1}}\left|E_{k}\right|^{\frac{1}{p}}\left|E_{j}\right|^{-\frac{1}{p}}\left\|\chi_{j} f\right\|_{L^{p}\left(\mathbb{R}^{n}\right)}
\end{aligned}
$$

Since $-\frac{1}{p}+\frac{\lambda}{q}<\theta<\left(1-\frac{1}{p}\right)+\frac{\lambda}{q}$, choosing $N$ large enough, we obtain

$$
\begin{aligned}
A_{3} \lesssim & \left(1+r m_{V}\left(x_{0}\right)\right)^{\alpha} r^{-\lambda n} \sum_{k=-\infty}^{0}\left|E_{k}\right|^{\theta q}\left(\sum_{j=k+2}^{\infty} \frac{\left|E_{k}\right|^{\frac{1}{p}}\left|E_{j}\right|^{-\frac{1}{p}}\left\|\chi_{j} f\right\|_{L^{p}\left(\mathbb{R}^{n}\right)}}{\left(1+2 j r m_{V}\left(x_{0}\right)\right)^{N / k_{0}+1}}\right)^{q} \\
\lesssim & \left(1+r m_{V}\left(x_{0}\right)\right)^{\alpha} r^{-\lambda n} \sum_{k=-\infty}^{0}\left|E_{k}\right|^{\theta q} \\
& \times\left\{\sum_{j=k+2}^{\infty} \frac{\left(1+2^{j} r m_{V}\left(x_{0}\right)\right)^{-\frac{\alpha}{q}}\left(2^{j} r\right)^{\frac{\lambda n}{q}}\left|E_{j}\right|^{-\alpha}}{\left(1+2^{j} r m_{V}\left(x_{0}\right)\right)^{N / k_{0}+1}}\right. \\
& \left.\times 2^{(k-j) \frac{n}{p}}\left(1+2^{j} r m_{V}\left(x_{0}\right)\right)^{\frac{\alpha}{q}}\left(2^{j} r\right)^{-\frac{\lambda n}{q}}\left(\left|E_{j}\right|^{\theta q}\left\|\chi_{j} f\right\|_{L^{p}\left(\mathbb{R}^{n}\right)}^{q}\right)^{\frac{1}{q}}\right\}^{q} \\
\lesssim & \left.\left(1+r m_{V}\left(x_{0}\right)\right)^{\alpha} r^{-\lambda n} \sum_{k=-\infty}^{0}\left|E_{k}\right|^{\theta q}\left(\sum_{j=k+2}^{\infty} 2^{(k-j) \frac{n}{p}\left|E_{j}\right|^{\frac{\lambda}{q}-\theta}}\right)^{q}\|f\|_{L_{\alpha, \theta, V}^{p, q, \lambda}}^{q} \mathbb{R}^{n}\right) \\
\lesssim & \|f\|_{L_{\alpha, \theta, V}^{p, q, \lambda}\left(\mathbb{R}^{n}\right)}^{q}
\end{aligned}
$$

Let $N=\left[-\frac{\alpha}{q}+1\right]\left(k_{0}+1\right)$. Finally, $\|T f\|_{L_{\alpha, \theta, V}^{p, q, \lambda}\left(\mathbb{R}^{n}\right)} \lesssim\|f\|_{L_{\alpha, \theta, V}^{p, q, \lambda}\left(\mathbb{R}^{n}\right)^{\prime}}$. This completes the proof of Theorem 3.2.

Suppose that $b \in B M O\left(\mathbb{R}^{n}\right)$ and $V \in B_{n}$. Let $T$ be one of the Schrödinger type operators $\nabla(-\Delta+V)^{-1} \nabla, \nabla(-\Delta+V)^{-1 / 2}$ and $(-\Delta+V)^{-1 / 2} \nabla$. The commutator $[b, T]$ is defined as

$$
[b, T] f=b T(f)-T(b f) .
$$

Theorem 3.3 Suppose that $V \in B_{n}$ and $b \in B M O\left(\mathbb{R}^{n}\right)$. Let $1<p<\infty, 1<q<\infty, \alpha \in$ $(-\infty, 0], \lambda \in(0, n)$. If the index $(p, q, \theta, \lambda)$ satisfies $\frac{\lambda}{q}-\frac{1}{p}<\theta<\frac{\lambda}{q}+1-\frac{1}{p}$, then

$$
\|[b, T] f\|_{L_{\alpha, \theta, V}^{p, q, \lambda}} \leq C\|f\|_{L_{\alpha, \theta, V}^{p, q, \lambda}}\|b\|_{B M O} .
$$

Proof For any ball $B=B\left(x_{0}, r\right)$, we can get

$$
f(y)=\sum_{j=-\infty}^{\infty} f(y) \chi_{E_{j}}(y)=\sum_{j=-\infty}^{\infty} f_{j}(y)
$$


where $E_{j}=B\left(x_{0}, 2^{j} r\right) \backslash B\left(x_{0}, 2^{j-1} r\right)$. Hence, we have

$$
\begin{aligned}
\left(1+r m_{V}\left(x_{0}\right)\right)^{\alpha} r^{-\lambda n} \sum_{k=-\infty}^{0}\left|E_{k}\right|^{\theta q}\left\|\chi_{k}[b, T] f\right\|_{L^{p}\left(\mathbb{R}^{n}\right)}^{q} \\
\lesssim\left(1+r m_{V}\left(x_{0}\right)\right)^{\alpha} r^{-\lambda n} \sum_{k=-\infty}^{0}\left|E_{k}\right|^{\theta q}\left(\sum_{j=-\infty}^{k-2}\left\|\chi_{k}[b, T] f_{j}\right\|_{L^{p}\left(\mathbb{R}^{n}\right)}\right)^{q} \\
\quad+\left(1+r m_{V}\left(x_{0}\right)\right)^{\alpha} r^{-\lambda n} \sum_{k=-\infty}^{0}\left|E_{k}\right|^{\theta q}\left(\sum_{j=k-1}^{k+1}\left\|\chi_{k}[b, T] f_{j}\right\|_{L^{p}\left(\mathbb{R}^{n}\right)}\right)^{q} \\
\quad+\left(1+r m_{V}\left(x_{0}\right)\right)^{\alpha} r^{-\lambda n} \sum_{k=-\infty}^{0}\left|E_{k}\right|^{\theta q}\left(\sum_{j=k+2}^{\infty}\left\|\chi_{k}[b, T] f_{j}\right\|_{L^{p}\left(\mathbb{R}^{n}\right)}\right)^{q} \\
=: \\
B_{1}+B_{2}+B_{3} .
\end{aligned}
$$

For $B_{2}$, by Corollary 2.11, we have

$$
\begin{aligned}
B_{2} & \lesssim\left(1+r m_{V}\left(x_{0}\right)\right)^{\alpha} r^{-\lambda n} \sum_{k=-\infty}^{0}\left|E_{k}\right|^{\theta q}\left(\sum_{j=k-1}^{k+1}\left\|[b, T] f_{j}\right\|_{L^{p}\left(\mathbb{R}^{n)}\right)}\right)^{q} \\
& \lesssim\left(1+r m_{V}\left(x_{0}\right)\right)^{\alpha} r^{-\lambda n} \sum_{k=-\infty}^{0}\left|E_{k}\right|^{\theta q}\left(\sum_{j=k-1}^{k+1}\left\|f_{j}\right\|_{L^{p}\left(\mathbb{R}^{n)}\right)}\right)^{q}\|b\|_{B M O}^{q} \\
& \lesssim\|f\|_{L_{\alpha, \theta, V}^{p, q, \lambda}}^{q}\|b\|_{B M O}^{q} .
\end{aligned}
$$

Denote by $b_{2^{k} r}$ the mean value of $b$ on the ball $B\left(x_{0}, 2^{k} r\right)$. For $B_{1}$, by Lemma 2.5 and (2.2), we have

$$
\begin{aligned}
\| \chi_{k}[ & b, T] f_{j} \|_{L^{p}\left(\mathbb{R}^{n}\right)} \\
\lesssim & \frac{1}{\left(1+2^{k} r m_{V}\left(x_{0}\right)\right)^{N / k_{0}+1}} \frac{1}{\left(2^{k} r\right)^{n}} \\
& \times\left[\int_{E_{k}}\left(\int_{E_{j}}|b(x)-b(y)| f(y) \mid d y\right)^{p} d x\right]^{\frac{1}{p}} \\
\lesssim & \frac{1}{\left(1+2^{k} r m_{V}\left(x_{0}\right)\right)^{N / k_{0}+1}} \frac{1}{\left(2^{k} r\right)^{n}}\left[\left(\int_{E_{k}}\left|b(x)-b_{2^{k}}\right|^{p} d x\right)^{\frac{1}{p}} \int_{E_{j}}|f(y)| d y\right. \\
& \left.+\left|E_{k}\right|^{\frac{1}{p}} \int_{E_{j}}\left|b(y)-b_{2^{k} r}\right||f(y)| d y\right] \\
\lesssim & \frac{1}{\left(1+2^{k} r m_{V}\left(x_{0}\right)\right)^{N / k_{0}+1}} \frac{1}{\left(2^{k} r\right)^{n}}\left[\left|E_{k}\right|^{\frac{1}{p}}\left|E_{j}\right|^{1-\frac{1}{p}}\|b\|_{B M O}\left\|f_{j}\right\|_{L^{p}\left(\mathbb{R}^{n}\right)}\right. \\
& +\left|E_{k}\right|^{\frac{1}{p}}\left\|f_{j}\right\|_{L^{p}}\left(\mathbb{R}^{n)}\left(\int_{E_{j}}\left|b(y)-b_{2^{k}}\right|^{p^{\prime}} d x\right)^{\frac{1}{p^{\prime}}}\right] \\
\lesssim & \frac{1}{\left(1+2^{k} r m_{V}\left(x_{0}\right)\right)^{N / k_{0}+1}} \frac{\left|E_{j}\right|^{1-\frac{1}{p}}}{\left|E_{k}\right|^{1-\frac{1}{p}}}(k-j)\left\|f_{j}\right\|_{L^{p}\left(\mathbb{R}^{n}\right)}\|b\|_{B M O},
\end{aligned}
$$


where in the third inequality, we have used John-Nirenberg's inequality [19]. Since $-\frac{1}{p}+\frac{\lambda}{q}<$ $\theta<\left(1-\frac{1}{p}\right)+\frac{\lambda}{q}$, we obtain

$$
\begin{aligned}
B_{1} \lesssim & \frac{\left(1+r m_{V}\left(x_{0}\right)\right)^{\alpha}}{r^{\lambda n}} \sum_{k=-\infty}^{0}\left|E_{k}\right|^{\theta q}\left(\sum_{j=-\infty}^{k-2} \frac{(k-j)\left\|f_{j}\right\|_{L^{p}}\left(\mathbb{R}^{n}\right)}{\left(1+2^{k} r m_{V}\left(x_{0}\right)\right)^{N / k_{0}+1}} \frac{\left|E_{j}\right|^{1-\frac{1}{p}}}{\left|E_{k}\right|^{1-\frac{1}{p}}}\right)^{q}\|b\|_{B M O}^{q} \\
\lesssim & \frac{\left(1+r m_{V}\left(x_{0}\right)\right)^{\alpha}}{r^{\lambda n}} \sum_{k=-\infty}^{0}\left|E_{k}\right|^{\theta q}\left[\sum_{j=-\infty}^{k-2} \frac{\left(1+2^{j} r m_{V}\left(x_{0}\right)\right)^{-\frac{\alpha}{q}}}{\left(1+2^{k} r m_{V}\left(x_{0}\right)\right)^{N / k_{0}+1}}\|b\|_{B M O}^{q}\right. \\
& \left.\times(k-j)\left(2^{j} r\right)^{\frac{\lambda n}{q}}\left|E_{j}\right|^{-\theta} \frac{\left|E_{j}\right|^{1-\frac{1}{p}}}{\left|E_{k}\right|^{1-\frac{1}{p}}}\right]^{q}\|f\|_{L_{\alpha, \theta, V}^{p, q, \lambda}}^{q} \\
\lesssim & \frac{\left(1+r m_{V}\left(x_{0}\right)\right)^{\alpha}}{r^{\lambda n}} \sum_{k=-\infty}^{0}\left|E_{k}\right|^{\lambda}\left(\sum_{j=-\infty}^{k-2}(k-j) 2^{(k-j) n\left(\theta-\frac{\alpha}{q}+\frac{1}{p}-1\right)}\right)^{q}\|f\|_{L_{\alpha, \theta, V}^{p, q, \lambda}}^{q}\|b\|_{B M O}^{q} \\
\lesssim & \|f\|_{L_{\alpha, \theta, V}^{p, q, \lambda}}^{q}\|b\|_{B M O}^{q} .
\end{aligned}
$$

For $B_{3}$, similar to $B_{1}$, we have

$$
\begin{aligned}
& \left\|\chi_{k}[b, T] f_{j}\right\|_{L^{p}\left(\mathbb{R}^{n}\right)} \\
& \quad \lesssim \frac{1}{\left(1+2 j r m_{V}\left(x_{0}\right)\right)^{N / k_{0}+1}} \frac{1}{(2 j r)^{n}}\left(\int_{E_{k}}\left|\int_{E_{j}}\right|(b(x)-b(y)) f(y)|d y|^{p} d x\right)^{\frac{1}{p}} \\
& \quad \lesssim \frac{j-k}{\left(1+2 j r m_{V}\left(x_{0}\right)\right)^{N / k_{0}+1}}\left|E_{k}\right|^{\frac{1}{p}}\left|E_{j}\right|^{-\frac{1}{p}}\left\|f_{j}\right\|_{L^{p}\left(\mathbb{R}^{n}\right)}\|b\|_{B M O} .
\end{aligned}
$$

Since $-\frac{1}{p}+\frac{\lambda}{q}<\theta<\left(1-\frac{1}{p}\right)+\frac{\lambda}{q}$, choosing $N$ large enough, we obtain

$$
\begin{aligned}
B_{3} \lesssim & \left(1+r m_{V}\left(x_{0}\right)\right)^{\alpha} r^{-\lambda n} \sum_{k=-\infty}^{0}\left|E_{k}\right|^{\theta q}\left(\sum_{j=k+2}^{\infty} \frac{\left|E_{k}\right|^{\frac{1}{p}}\left|E_{j}\right|^{-\frac{1}{p}}(j-k)\left\|f_{j}\right\|_{L^{p}\left(\mathbb{R}^{n}\right)}}{\left(1+2^{j} r m_{V}\left(x_{0}\right)\right)^{N / k_{0}+1}}\right)^{q}\|b\|_{B M O}^{q} \\
\lesssim & \left(1+r m_{V}\left(x_{0}\right)\right)^{\alpha} r^{-\lambda n} \sum_{k=-\infty}^{0}\left|E_{k}\right|^{\theta q}\left[\sum_{j=k+2}^{\infty} \frac{\left(1+2^{j} r m_{V}\left(x_{0}\right)\right)^{-\frac{\alpha}{q}}}{\left(1+2^{j} r m_{V}\left(x_{0}\right)\right)^{N / k_{0}+1}}\right. \\
& \left.\times(j-k)\left(2^{j} r\right)^{\frac{\lambda n}{q}}\left|E_{j}\right|^{-\theta}\left|E_{k}\right|^{\frac{1}{p}}\left|E_{j}\right|^{-\frac{1}{p}}\right]^{q}\|f\|_{L_{\alpha, \theta, V}^{p, q, \lambda}\left(\mathbb{R}^{n}\right)}^{q}\|b\|_{B M O}^{q} \\
\lesssim & \left(1+r m_{V}\left(x_{0}\right)\right)^{\alpha} r^{-\lambda n} \sum_{k=-\infty}^{0}\left|E_{k}\right|^{\lambda}\left(\sum_{j=k+2}^{\infty} 2^{(k-j) n\left(\frac{1}{p}-\frac{\lambda}{q}+\theta\right)}\right)^{q}\|f\|_{L_{\alpha, \theta, V}^{p, q, \lambda}\left(\mathbb{R}^{n}\right)}^{q}\|b\|_{B M O}^{q} \\
\lesssim & \|f\|_{L_{\alpha, \theta, V}^{p, q, \lambda}\left(\mathbb{R}^{n}\right)}^{q}\|b\|_{B M O}^{q} .
\end{aligned}
$$

Let $N=\left[-\frac{\alpha}{q}+1\right]\left(k_{0}+1\right)$. We finally get

$$
\|[b, T] f\|_{L_{\alpha, \theta, V}^{p, q, \lambda}\left(\mathbb{R}^{n)}\right.} \lesssim\|f\|_{L_{\alpha, \theta, V}^{p, q, \lambda}\left(\mathbb{R}^{n)}\right.}\|b\|_{B M O}
$$




\section{Schrödinger type operators on $L_{\alpha, \theta, V}^{p, q, \lambda}\left(\mathbb{R}^{n}\right)$}

Let $L=-\Delta+V$ be the Schrödinger operator, where $V \in B_{s}, s>n / 2$. For $0<\beta<\frac{n}{2}$, the fractional integral operator associated with $L$ is defined by

$$
L^{-\beta}(f)(x)=\int_{0}^{\infty} e^{-t L}(f)(x) t^{\beta-1} d t
$$

Denote by $K_{\beta}(x, y)$ the kernel of $L^{-\beta}$. By Lemma 2.12, Bui [20] obtained the following pointwise estimate.

Lemma 4.1 (Proposition 3.3 in [20]) Let $0<\beta<\frac{n}{2}$. For $N \in \mathbb{N}$, there is a constant $C_{N}$ such that

$$
\begin{aligned}
K_{\beta}(x, y) & =\int_{0}^{\infty} K_{t}(x, y) t^{\beta-1} d t \\
& \leq \frac{C_{N}}{\left(1+|x-y| m_{V}(x)\right)^{N}} \frac{1}{|x-y|^{n-2 \beta}},
\end{aligned}
$$

where $K_{t}(\cdot, \cdot)$ is the kernel of the semigroup $e^{-t L}$.

Definition 4.2 Let $f \in L_{\text {loc }}^{q}\left(\mathbb{R}^{n}\right)$. Denote by $|B|$ the Lebesgue measure of the ball $B \subset \mathbb{R}^{n}$. The fractional Hardy-Littlewood maximal function $M_{\sigma, \gamma}$ is defined by

$$
M_{\sigma, \gamma} f(x)=\sup _{x \in B}\left(\frac{1}{|B|^{1-\frac{\sigma \gamma}{n}}} \int_{B}|f(y)|^{\gamma} d y\right)^{\frac{1}{\gamma}}
$$

Lemma 4.3 ([16]) Suppose $1<\gamma<p_{1}<\frac{n}{\sigma}$ and $\frac{1}{p_{2}}=\frac{1}{p_{1}}-\frac{\sigma}{n}$. Then

$$
\left\|M_{\sigma, \gamma} f\right\|_{L^{p_{2}\left(\mathbb{R}^{n}\right)}} \lesssim\|f\|_{L^{p_{1}\left(\mathbb{R}^{n}\right)}} .
$$

As a generalization of the fractional integral associated with $L$, the operators $V^{\beta_{2}}(-\Delta+$ $V)^{-\beta_{1}}, 0 \leq \beta_{2} \leq \beta_{1} \leq 1$, have been studied by Sugano [9] systematically. Applying the method of Sugano [9] together with Lemma 4.1, we can obtain the following result for $V^{\beta_{2}}(-\Delta+V)^{-\beta_{1}}, 0 \leq \beta_{2} \leq \beta_{1} \leq n / 2$. We omit the proof.

Theorem 4.4 Suppose that $V \in B_{\infty}$. Let $1<\beta_{2} \leq \beta_{1}<\frac{n}{2}$. Then

$$
\left|V^{\beta_{2}}(-\Delta+V)^{-\beta_{1}} f(x)\right| \lesssim M_{2\left(\beta_{1}-\beta_{2}\right), 1} f(x) .
$$

In a similar way, by (4.1), we can get the following estimate for the operators $(-\Delta+$ $V)^{-\beta_{1}} V^{\beta_{2}}, 0 \leq \beta_{2} \leq \beta_{1}<\frac{n}{2}$.

Theorem 4.5 Suppose that $V \in B_{s}$ for $s>\frac{n}{2}$. Let $0 \leq \beta_{2} \leq \beta_{1}<\frac{n}{2}$. Then

$$
\left|(-\Delta+V)^{-\beta_{1}}\left(V^{\beta_{2}} f\right)(x)\right| \lesssim M_{2\left(\beta_{1}-\beta_{2}\right)}(f)(x),
$$

where $\left(\frac{s}{\beta_{2}}\right)^{\prime}$ is the conjugate of $\left(\frac{s}{\beta_{2}}\right)$. 
Proof Let $r=1 / m_{V}(x)$. By Lemma 4.1 and Hölder's inequality, we have

$$
\begin{aligned}
\mid(- & \Delta+V)^{-\beta_{1}} V^{\beta_{2}}(x) f(x) \mid \\
& \lesssim \sum_{k=-\infty}^{\infty} \int_{2^{k-1} r \leq|x-y| \leq 2^{k_{r}}} \frac{1}{\left(1+2^{k} r m_{V}\left(x_{0}\right)\right)^{N}} \frac{1}{\left(2^{k} r\right)^{n-2 \beta_{1}}} V(y)^{\beta_{2}}|f(y)| d y \\
& \lesssim \sum_{k=-\infty}^{\infty} \frac{\left(2^{k} r\right)^{2 \beta_{2}}}{\left(1+2^{k}\right)^{N}}\left(\frac{1}{\left(2^{k} r\right)^{n}} \int_{B\left(x, 2^{k} r\right)} V(y) d y\right)^{\beta_{2}} M_{2\left(\beta_{1}-\beta_{2}\right),\left(\frac{s}{\beta_{2}}\right)^{\prime}}(f)(x) .
\end{aligned}
$$

For $k \geq 1$, because $V(y) d y$ is a doubling measure, we have

$$
\begin{aligned}
\frac{\left(2^{k} r\right)^{2}}{\left(2^{k} r\right)^{n}} \int_{B\left(x, 2^{k} r\right)} V(y) d y & \lesssim C_{0}^{k} \cdot 2^{(2-n) k} \frac{r^{2}}{r^{n}} \int_{B(x, r)} V(y) d y \\
& \lesssim\left(2^{k}\right)^{k_{0}}
\end{aligned}
$$

where $k_{0}=2-n+\log _{2} C_{0}$. For $k \leq 0$, Lemma 2.6 implies that

$$
\begin{aligned}
\frac{\left(2^{k} r\right)^{2}}{\left(2^{k} r\right)^{n}} \int_{B\left(x, 2^{k} r\right)} V(y) d y & \lesssim\left(\frac{r}{2^{k} r}\right)^{\frac{n}{s}-2} \frac{r^{2}}{r^{n}} \int_{B(x, r)} V(y) d y \\
& \lesssim\left(2^{k}\right)^{2-\frac{n}{s}}
\end{aligned}
$$

Taking $N$ large enough, we get

$$
\left|(-\Delta+V)^{-\beta_{1}} V^{\beta_{2}} f(x)\right| \lesssim M_{2\left(\beta_{1}-\beta_{2}\right),\left(\frac{s}{\beta_{2}}\right)^{\prime}} f(x)
$$

By Theorem 4.5 and the duality, we can obtain the following.

Corollary 4.6 Suppose $V \in B_{s}$ for $s>\frac{n}{2}$.

(1) If $1<\left(\frac{s}{\beta_{2}}\right)^{\prime}<p_{1}<\frac{n}{2 \beta_{1}-2 \beta_{2}}$ and $\frac{1}{p_{2}}=\frac{1}{p_{1}}-\frac{2 \beta_{1}-2 \beta_{2}}{n}$, then

$$
\left\|(-\Delta+V)^{-\beta_{1}} V^{\beta_{2}} f\right\|_{L^{p_{2}\left(\mathbb{R}^{n}\right)}} \lesssim\|f\|_{L^{p_{1}\left(\mathbb{R}^{n}\right)}},
$$

where $\frac{s}{\beta_{2}}+\left(\frac{s}{\beta_{2}}\right)^{\prime}=1$.

(2) If $1<p_{2}<\frac{s}{\beta_{2}}$ and $\frac{1}{p_{2}}=\frac{1}{p_{1}}-\frac{2 \beta_{1}-2 \beta_{2}}{n}$, then

$$
\left\|V^{\beta_{2}}(-\Delta+V)^{-\beta_{1}} f\right\|_{L^{p_{2}\left(\mathbb{R}^{n}\right)}} \lesssim\|f\|_{L^{p_{1}\left(\mathbb{R}^{n}\right)}} .
$$

Theorem 4.7 Suppose that $V \in B_{s}, s \geq \frac{n}{2}, \alpha \in(-\infty, 0], \lambda \in(0, n)$. Let $1<q<\infty, 1<\beta_{2} \leq$ $\beta_{1}<\frac{n}{2}$ and $1<p_{2}<\frac{s}{\beta_{2}}$ with $\frac{1}{p_{1}}-\frac{1}{p_{2}}=\frac{2 \beta_{1}-2 \beta_{2}}{n}$. If $\frac{\lambda}{q}-\frac{1}{p_{1}}+\frac{2 \beta_{1}}{n}<\theta<\frac{\lambda}{q}+1-\frac{1}{p_{1}}$, then

$$
\left\|V^{\beta_{2}}(-\Delta+V)^{-\beta_{1}} f\right\|_{L_{\alpha, \theta, V}^{p_{2}, q, \lambda}} \lesssim\|f\|_{L_{\alpha, \theta, V}^{p_{1}, q, \lambda}}
$$

Proof For any ball $B\left(x_{0}, r\right)$, write

$$
f(y)=\sum_{j=-\infty}^{\infty} f(y) \chi_{E_{j}}(y)=\sum_{j=-\infty}^{\infty} f_{j}(y)
$$


where $E_{j}=B\left(x_{0}, 2^{j} r\right) \backslash B\left(x_{0}, 2^{j-1} r\right)$. Hence, we have

$$
\begin{aligned}
& \left(1+r m_{V}\left(x_{0}\right)\right)^{\alpha} r^{-\lambda n} \sum_{k=-\infty}^{0}\left|E_{k}\right|^{\theta q}\left\|\chi_{k} V^{\beta_{2}}(-\Delta+V)^{-\beta_{1}} f\right\|_{L^{p_{2}\left(\mathbb{R}^{n}\right)}}^{q} \\
& \lesssim\left(1+r m_{V}\left(x_{0}\right)\right)^{\alpha} r^{-\lambda n} \sum_{k=-\infty}^{0}\left|E_{k}\right|^{\theta q}\left(\sum_{j=-\infty}^{k-2}\left\|\chi_{k} V^{\beta_{2}}(-\Delta+V)^{-\beta_{1}} f_{j}\right\|_{L^{p_{2}\left(\mathbb{R}^{n}\right)}}\right)^{q} \\
& \quad+\left(1+r m_{V}\left(x_{0}\right)\right)^{\alpha} r^{-\lambda n} \sum_{k=-\infty}^{0}\left|E_{k}\right|^{\theta q}\left(\sum_{j=k-1}^{k+1}\left\|\chi_{k} V^{\beta_{2}}(-\Delta+V)^{-\beta_{1}} f_{j}\right\|_{\left.L^{p_{2}\left(\mathbb{R}^{n}\right)}\right)^{q}}\right. \\
& \quad+\left(1+r m_{V}\left(x_{0}\right)\right)^{\alpha} r^{-\lambda n} \sum_{k=-\infty}^{0}\left|E_{k}\right|^{\theta q}\left(\sum_{j=k+2}^{\infty}\left\|\chi_{k} V^{\beta_{2}}(-\Delta+V)^{-\beta_{1}} f_{j}\right\|_{\left.L^{p_{2}\left(\mathbb{R}^{n}\right)}\right)^{q}}\right. \\
& =M_{1}+M_{2}+M_{3} .
\end{aligned}
$$

We first estimate $M_{2}$. For $1<p_{2}<\frac{s}{\beta_{2}}$, by (2) of Corollary 4.6, we can get

$$
M_{2} \lesssim \frac{\left(1+r m_{V}\left(x_{0}\right)\right)^{\alpha}}{r^{\lambda n}} \sum_{k=-\infty}^{0}\left|E_{k}\right|^{\theta q}\left(\sum_{j=k-1}^{k+1}\left\|f_{j}\right\|_{L^{p_{1}}\left(\mathbb{R}^{n}\right)}\right)^{q} \lesssim\|f\|_{L_{\alpha, \theta, V}^{q}}^{q}
$$

Now we deal with the terms $M_{1}$ and $M_{3}$. We choose $N$ large enough such that

$$
\left(N / k_{0}+1\right)-\left(\log _{2} C_{0}+1\right) \beta_{2}+\alpha / q>0
$$

and take positive $N_{1}<\left(N / k_{0}+1\right)-\left(\log _{2} C_{0}+1\right) \beta_{2}$. For $M_{1}$, note that if $x \in E_{k}, y \in E_{j}$ and $j \leq k-2$, then $|x-y| \sim 2^{k}$. By Lemmas 4.1 and 2.7 , we use Hölder's inequality to obtain

$$
\begin{aligned}
&\left\|\chi_{k} V^{\beta_{2}}(-\Delta+V)^{-\beta_{1}} f_{j}\right\|_{L^{p_{2}}\left(\mathbb{R}^{n}\right)} \\
& \quad \lesssim\left(\int_{E_{k}}\left|V^{\beta_{2}}(x) \int_{E_{j}} \frac{1}{\left(1+|x-y| m_{v}(x)\right)^{N}} \frac{1}{|x-y|^{n-2 \beta_{1}}} f(y) d y\right|^{p_{2}} d x\right)^{\frac{1}{p_{2}}} \\
& \lesssim \frac{1}{\left(1+2^{k} r m_{V}\left(x_{0}\right)\right)^{N / k_{0}+1}} \frac{1}{\left(2^{k} r\right)^{n-2 \beta_{1}}} \int_{E_{j}}|f(y)| d y\left(\int_{E_{k}}|V(x)|^{\beta_{2} p_{2}} d x\right)^{\frac{1}{p_{2}}} \\
& \lesssim \frac{\left|E_{j}\right|^{1-\frac{1}{p_{1}}}\left|E_{k}\right|^{\frac{1}{p_{2}}}}{\left(1+2^{k} r m_{V}\left(x_{0}\right)\right)^{N / k_{0}+1}} \frac{1}{\left(2^{k} r\right)^{n-2 \beta_{1}}} \mid f_{j} \|_{L^{p_{1}\left(\mathbb{R}^{n}\right)}}\left(\frac{1}{\left|E_{k}\right|} \int_{E_{k}} V(x)^{s} d x\right)^{\frac{\beta_{2}}{s}} \\
& \lesssim \frac{\left|E_{j}\right|^{1-\frac{1}{p_{1}}}\left|E_{k}\right|^{\frac{1}{p_{2}}}}{\left(1+2^{k} r m_{V}\left(x_{0}\right)\right)^{N / k_{0}+1}} \frac{1}{\left(2^{k} r\right)^{n-2 \beta_{1}}} \mid f_{j} \|_{L^{p_{1}}\left(\mathbb{R}^{n}\right)}\left(\frac{1}{\left|B_{k}\right|} \int_{B_{k}} V(x) d x\right)^{\beta_{2}} \\
& \lesssim \frac{1}{\left(1+2^{k} r m_{V}\left(x_{0}\right)\right)^{N_{1}}} \frac{1}{\left(2^{k} r\right)^{n-2 \beta_{1}+2 \beta_{2}}}\left|E_{k}\right|^{\frac{1}{p_{2}}}\left|E_{j}\right|^{1-\frac{1}{p_{1}}}\left\|f_{j}\right\|_{L^{p_{1}\left(\mathbb{R}^{n}\right)},}
\end{aligned}
$$

where $\frac{1}{p_{1}}-\frac{1}{p_{2}}=\frac{2 \beta_{1}-2 \beta_{2}}{n}$. Since $\frac{\lambda}{q}-\frac{1}{p_{1}}+\frac{2 \beta_{1}}{n}<\theta<\frac{\lambda}{q}+1-\frac{1}{p_{1}}$, we obtain

$$
\begin{aligned}
M_{1} \lesssim & \left(1+r m_{V}\left(x_{0}\right)\right)^{\alpha} r^{-\lambda n} \sum_{k=-\infty}^{0}\left|E_{k}\right|^{\theta q} \\
& \times\left(\sum_{j=-\infty}^{k-2} \frac{1}{\left(1+2^{k} r m_{V}\left(x_{0}\right)\right)^{N_{1}}} \frac{1}{\left(2^{k} r\right)^{n-2 \beta_{1}+2 \beta_{2}}}\left|E_{k}\right|^{\frac{1}{p_{2}}}\left|E_{j}\right|^{1-\frac{1}{p_{1}}}\left\|f_{j}\right\|_{\left.L^{p_{1}\left(\mathbb{R}^{n}\right)}\right)^{q}}^{q}\right.
\end{aligned}
$$




$$
\begin{aligned}
& \lesssim\left(1+r m_{V}\left(x_{0}\right)\right)^{\alpha} r^{-\lambda n} \sum_{k=-\infty}^{0}\left|E_{k}\right|^{\theta q} \\
& \times\left(\sum_{j=-\infty}^{k-2} \frac{\left(1+2^{j} r m_{V}\left(x_{0}\right)\right)^{-\frac{\alpha}{q}}}{\left(1+2^{k} r m_{V}\left(x_{0}\right)\right)^{N_{1}}} \frac{\left(2^{j} r\right)^{\frac{\lambda n}{q}}\left|E_{j}\right|^{-\theta}}{\left(2^{k} r\right)^{n-2 \beta_{1}+2 \beta_{2}}}\left|E_{k}\right|^{\frac{1}{p_{2}}}\left|E_{j}\right|^{1-\frac{1}{p_{1}}}\right)^{q}\|f\|_{L_{\alpha, \nu, \theta}^{p_{1}, \lambda, q}}^{q} \\
& \lesssim\left(1+r m_{V}\left(x_{0}\right)\right)^{\alpha} r^{-\lambda n} \sum_{k=-\infty}^{0}\left|E_{k}\right|^{\lambda}\left(\sum_{j=-\infty}^{k-2} 2^{(j-k) n\left(\frac{\lambda}{q}-\theta-\frac{1}{p_{1}}+1\right)}\right)^{q}\|f\|_{L_{\alpha, \nu, \theta}^{p_{1}, \lambda, q}}^{q} \\
& \lesssim\|f\|_{L_{\alpha, \nu, \theta}^{p_{1}, \lambda, q}}^{q}
\end{aligned}
$$

For $M_{3}$, note that when $x \in E_{k}, y \in E_{j}$ and $j \geq k+2$, then $|x-y| \sim 2^{j} r$. Similar to $E_{1}$, we have

$$
\begin{aligned}
& \left\|\chi_{k} V^{\beta_{2}}(-\Delta+V)^{-\beta_{1}} f_{j}\right\|_{L^{p_{2}}\left(\mathbb{R}^{n}\right)} \\
& \quad \lesssim \frac{1}{\left(1+2^{j} r m_{V}\left(x_{0}\right)\right)^{N / k_{0}+1}} \frac{1}{(2 j r)^{n-2 \beta_{1}}} \int_{E_{j}}|f(y)| d y\left(\int_{E_{k}}|V(x)|^{\beta_{2} p_{2}} d x\right)^{\frac{1}{p_{2}}} \\
& \quad \lesssim \frac{1}{\left(1+2^{j} r m_{V}\left(x_{0}\right)\right)^{N_{1}}}\left|E_{j}\right|^{\frac{2 \beta_{1}}{n}-\frac{1}{p_{1}}}\left|E_{k}\right|^{\frac{1}{p_{2}}-\frac{2 \beta_{2}}{n}} \mid f_{j} \|_{L^{p_{1}}\left(\mathbb{R}^{n}\right)},
\end{aligned}
$$

where $\frac{1}{p_{1}}-\frac{1}{p_{2}}=\frac{2 \beta_{1}-2 \beta_{2}}{n}$. Since $\frac{\lambda}{q}-\frac{1}{p_{1}}+\frac{2 \beta_{1}}{n}<\theta<\frac{\lambda}{q}+1-\frac{1}{p_{1}}$, we obtain

$$
\begin{aligned}
M_{3} \lesssim & \left(1+r m_{V}\left(x_{0}\right)\right)^{\alpha} r^{-\lambda n} \sum_{k=-\infty}^{0}\left|E_{k}\right|^{\theta q} \\
& \times\left(\sum_{j=k+2}^{\infty} \frac{1}{\left(1+2^{j} r m_{V}\left(x_{0}\right)\right)^{N_{1}}}\left|E_{j}\right|^{\frac{2 \beta_{1}}{n}-\frac{1}{p_{1}}}\left|E_{k}\right|^{\frac{1}{p_{2}}-\frac{2 \beta_{2}}{n}}\left\|f_{j}\right\|_{L^{p_{1}}\left(\mathbb{R}^{n}\right)}\right)^{q} \\
\lesssim & \left(1+r m_{V}\left(x_{0}\right)\right)^{\alpha} r^{-\lambda n} \sum_{k=-\infty}^{0}\left|E_{k}\right|^{\theta q} \\
& \times\left(\sum_{j=k+2}^{\infty} \frac{\left(1+2^{j} r m_{V}\left(x_{0}\right)\right)^{-\frac{\alpha}{q}}\left(2^{j} r\right)^{\frac{\lambda n}{q}}\left|E_{j}\right|^{-\theta}}{\left(1+2^{j} r m_{V}\left(x_{0}\right)\right)^{N_{1}}} \frac{\left|E_{k}\right|^{\frac{1}{p_{2}}-\frac{2 \beta_{2}}{n}}}{\left|E_{j}\right|^{\frac{2 \beta_{1}}{n}-\frac{1}{p_{1}}}}\right)^{q}\|f\|_{L_{\alpha, \theta, V}^{p_{1}, q, \lambda}}^{q} \\
\lesssim & \left(1+r m_{V}\left(x_{0}\right)\right)^{\alpha} r^{-\lambda n} \sum_{k=-\infty}^{0}\left|E_{k}\right|^{\lambda}\left(\sum_{j=k+2}^{\infty} 2^{(k-j) n\left(\theta-\frac{\lambda}{q}+\frac{1}{p_{1}}+\frac{2 \beta_{1}}{n}\right)}\right)^{q}\|f\|_{L_{\alpha, \theta, V}^{p_{1}, q, \lambda}}^{q} \\
\lesssim & \|f\|_{L_{\alpha, \theta, V}^{p_{1}, q, \lambda}}^{q .}
\end{aligned}
$$

Choosing $N$ large enough, we obtain

$$
\left\|V^{\beta_{2}}(-\Delta+V)^{-\beta_{1}} f\right\|_{L_{\alpha, \theta, V}^{p_{2}, q, \lambda}} \lesssim\|f\|_{L_{\alpha, \theta, V}^{p_{1}, q, \lambda}}
$$

Theorem 4.8 Suppose that $V \in B_{s}, s \geq \frac{n}{2}, \alpha \in(-\infty, 0], \lambda \in(0, n)$ and $1<q<\infty$. Let $0<$ $\beta_{2} \leq \beta_{1}<\frac{n}{2}, \frac{s}{s-\beta_{2}}<p_{1}<\frac{n}{2 \beta_{1}-2 \beta_{2}}$ with $\frac{1}{p_{2}}=\frac{1}{p_{1}}-\frac{2 \beta_{1}-2 \beta_{2}}{n}$. If $\frac{\lambda}{q}-\frac{1}{p_{2}}<\theta<\frac{\lambda}{q}-\frac{1}{p_{2}}+1-\frac{2 \beta_{1}}{n}$, then

$$
\left\|(-\Delta+V)^{-\beta_{1}} V^{\beta_{2}} f\right\|_{L_{\alpha, \theta, V}^{p_{2}, q, \lambda}} \lesssim\|f\|_{L_{\alpha, \theta, V}^{p_{1}, q, \lambda}}
$$


Proof For any ball $B\left(x_{0}, r\right)$, let $E_{j}=B\left(x_{0}, 2^{j} r\right) \backslash B\left(x_{0}, 2^{j-1} r\right)$. We can decompose $f$ as follows:

$$
f(y)=\sum_{j=-\infty}^{\infty} f(y) \chi_{E_{j}}(y)=\sum_{j=-\infty}^{\infty} f_{j}(y) .
$$

Similar to the proof of Theorem 4.7, we have

$$
\begin{aligned}
& \left(1+r m_{V}\left(x_{0}\right)\right)^{\alpha} r^{-\lambda n} \sum_{k=-\infty}^{0}\left|E_{k}\right|^{\theta q}\left\|\chi_{k}(-\Delta+V)^{-\beta_{1}} V^{\beta_{2}} f\right\|_{L^{p_{2}\left(\mathbb{R}^{n}\right)}}^{q} \\
& \lesssim\left(1+r m_{V}\left(x_{0}\right)\right)^{\alpha} r^{-\lambda n} \sum_{k=-\infty}^{0}\left|E_{k}\right|^{\theta q}\left(\sum_{j=-\infty}^{k-2}\left\|\chi_{k}(-\Delta+V)^{-\beta_{1}} V^{\beta_{2}} f_{j}\right\|_{L^{p_{2}\left(\mathbb{R}^{n}\right)}}\right)^{q} \\
& \quad+C\left(1+r m_{V}\left(x_{0}\right)\right)^{\alpha} r^{-\lambda n} \sum_{k=-\infty}^{0}\left|E_{k}\right|^{\theta q}\left(\sum_{j=k-1}^{k+1}\left\|\chi_{k}(-\Delta+V)^{-\beta_{1}} V^{\beta_{2}} f_{j}\right\|_{L^{p_{2}\left(\mathbb{R}^{n}\right)}}\right)^{q} \\
& \quad+C\left(1+r m_{V}\left(x_{0}\right)\right)^{\alpha} r^{-\lambda n} \sum_{k=-\infty}^{0}\left|E_{k}\right|^{\theta q}\left(\sum_{j=k+2}^{\infty}\left\|\chi_{k}(-\Delta+V)^{-\beta_{1}} V^{\beta_{2}} f_{j}\right\|_{\left.L^{p_{2}\left(\mathbb{R}^{n}\right)}\right)}\right)^{q} \\
& =L_{1}+L_{2}+L_{3} .
\end{aligned}
$$

For $L_{2}$, because $1<\frac{s}{s-\beta_{2}}<p_{1}<\frac{n}{2 \beta_{1}-\beta_{2}}$, we use Corollary 4.6 to obtain

$$
L_{2} \lesssim \frac{\left(1+r m_{V}\left(x_{0}\right)\right)^{\alpha}}{r^{\lambda n}} \sum_{k=-\infty}^{0}\left|E_{k}\right|^{\theta q}\left(\sum_{j=k-1}^{k+1}\left\|f_{j}\right\|_{L^{p_{1}}\left(\mathbb{R}^{n}\right)}\right)^{q} \lesssim\|f\|_{L_{\alpha, \theta, V}^{p_{1}, q_{\lambda} .}}^{q} .
$$

For $L_{1}$, we can see that if $x \in E_{k}$ and $y \in E_{j}$, then $|x-y| \sim 2^{k} r$ for $j \leq k-2$. By Hölder's inequality and the fact that $V \in B_{s}$, we deduce from Lemmas 4.1 and 2.7 that

$$
\begin{aligned}
& \left\|\chi_{k}(-\Delta+V)^{-\beta_{1}} V^{\beta_{2}} f_{j}\right\|_{L^{p_{2}}\left(\mathbb{R}^{n}\right)}^{q} \\
& \lesssim \frac{1}{\left(1+2^{k} r m_{V}\left(x_{0}\right)\right)^{N / k_{0}+1}} \frac{\left|E_{k}\right|^{\frac{1}{p_{2}}}}{\left(2^{k} r\right)^{n-2 \beta_{1}}} \int_{E_{j}} V(x)^{\beta_{2}}|f(y)| d y \\
& \lesssim \frac{1}{\left(1+2^{k} r m_{V}\left(x_{0}\right)\right)^{N / k_{0}+1}} \frac{\left|E_{k}\right|^{\frac{1}{p_{2}}}}{\left(2^{k} r\right)^{n-2 \beta_{1}}}\left|E_{j}\right|^{1-\frac{1}{p_{1}}}\left(\frac{1}{\left|B_{j}\right|} \int_{B_{j}} V(x) d x\right)^{\beta_{2}}\left\|f_{j}\right\|_{L^{p_{1}}\left(\mathbb{R}^{n}\right)} \\
& \lesssim \frac{1}{\left(1+2^{k} r m_{V}\left(x_{0}\right)\right)^{N_{2}}} \frac{\left|E_{k}\right|^{\frac{1}{p_{2}}}}{\left(2^{k} r\right)^{n-2 \beta_{1}}}\left|E_{j}\right|^{1-\frac{1}{p_{1}}}\left(2^{j} r\right)^{-2 \beta_{2}}\left\|f_{j}\right\|_{L^{p_{1}}\left(\mathbb{R}^{n}\right)},
\end{aligned}
$$

where $\frac{1}{p_{2}}=\frac{1}{p_{1}}-\frac{2 \beta_{1}-2 \beta_{2}}{n}$ and $N_{2}<\left(N / k_{0}+1\right)-\left(\log _{2} C_{0}+1\right) \beta_{2}$. Since $\frac{\lambda}{q}-\frac{1}{p_{2}}<\theta<\frac{\lambda}{q}-\frac{1}{p_{2}}+$ $1-\frac{2 \beta_{1}}{n}$, we obtain

$$
\begin{aligned}
L_{1} \lesssim & \left(1+r m_{V}\left(x_{0}\right)\right)^{\alpha} r^{-\lambda n} \sum_{k=-\infty}^{0}\left|E_{k}\right|^{\theta q} \\
& \times\left(\sum_{j=-\infty}^{k-2} \frac{1}{\left(1+2^{k} r m_{V}\left(x_{0}\right)\right)^{N_{2}}} \frac{\left|E_{k}\right|^{\frac{1}{p_{2}}}}{\left(2^{k} r\right)^{n-2 \beta_{1}}}\left|E_{j}\right|^{1-\frac{1}{p_{1}}}\left(2^{j} r\right)^{-2 \beta_{2}}\left\|f_{j}\right\|_{L^{p_{1}}\left(\mathbb{R}^{n}\right)}\right)^{q}
\end{aligned}
$$




$$
\begin{aligned}
\lesssim & \left(1+r m_{V}\left(x_{0}\right)\right)^{\alpha} r^{-\lambda n} \sum_{k=-\infty}^{0}\left|E_{k}\right|^{\theta q} \\
& \times\left(\sum_{j=-\infty}^{k-2} \frac{\left(1+2^{j} r m_{V}\left(x_{0}\right)\right)^{-\frac{\alpha}{q}}}{\left(1+2^{k} r m_{V}\left(x_{0}\right)\right)^{N_{2}}} \frac{\left(2^{j} r\right)^{\frac{\lambda n}{q}}\left|E_{j}\right|^{-\theta}}{\left(2^{k} r\right)^{n-2 \beta_{1}}} \frac{\left|E_{k}\right|^{\frac{1}{p_{2}}}\left|E_{j}\right|^{1-\frac{1}{p_{1}}}}{\left(2^{j} r\right)^{2 \beta_{2}}}\right)^{q}\|f\|_{L_{\alpha, V, \theta}^{p_{1}, \lambda, q}}^{q} \\
\lesssim & \left(1+r m_{V}\left(x_{0}\right)\right)^{\alpha} r^{-\lambda n} \sum_{k=-\infty}^{0}\left|E_{k}\right|^{\lambda}\left(\sum_{j=-\infty}^{k-2} 2^{(k-j) n\left(\theta-\frac{\lambda}{q}+\frac{1}{p_{2}}-1+\frac{2 \beta_{1}}{n}\right)}\right)^{q}\|f\|_{L_{\alpha, \theta, V}^{p_{1}, \lambda, q}}^{q} \\
\lesssim & \|f\|_{L_{\alpha, V, \theta}^{p_{1}, q, \lambda}}^{q .}
\end{aligned}
$$

For $L_{3}$, note that when $x \in E_{k}, y \in E_{j}$ and $j \geq k+2$, then $|x-y| \sim 2^{j} r$. Similar to $E_{1}$, we have

$$
\begin{aligned}
& \left\|\chi_{k}(-\Delta+V)^{-\beta_{1}} V^{\beta_{2}} f_{j}\right\|_{L^{p_{2}}\left(\mathbb{R}^{n}\right)}^{q} \\
& \lesssim \frac{1}{\left(1+2 j r m_{V}\left(x_{0}\right)\right)^{N / k_{0}+1}} \frac{\left|E_{k}\right|^{\frac{1}{p_{2}}}}{(2 j r)^{n-2 \beta_{1}}} \int_{E_{j}} V(x)^{\beta_{2}}|f(y)| d y \\
& \lesssim \frac{1}{\left(1+2 j r m_{V}\left(x_{0}\right)\right)^{N_{2}}} \\
& \frac{\left|E_{k}\right|^{\frac{1}{p_{2}}}}{(2 j r)^{n-2 \beta_{1}}}\left|E_{j}\right|^{1-\frac{1}{p_{1}}}\left(2^{j} r\right)^{-2 \beta_{2}}\left\|f_{j}\right\|_{L^{p_{1}}\left(\mathbb{R}^{n}\right)},
\end{aligned}
$$

where $\frac{1}{p_{2}}=\frac{1}{p_{1}}-\frac{2 \beta_{1}-2 \beta_{2}}{n}$ and $N_{2}<\left(N / k_{0}+1\right)-\left(\log _{2} C_{0}+1\right) \beta_{2}$. Since $\frac{\lambda}{q}-\frac{1}{p_{2}}<\theta<\frac{\lambda}{q}-\frac{1}{p_{2}}+$ $1-\frac{2 \beta_{1}}{n}$, we obtain

$$
\begin{aligned}
& L_{3} \lesssim\left(1+r m_{V}\left(x_{0}\right)\right)^{\alpha} r^{-\lambda n} \sum_{k=-\infty}^{0}\left|E_{k}\right|^{\theta q} \\
& \times\left(\sum_{j=k+2}^{\infty} \frac{1}{\left(1+2 j r m_{V}\left(x_{0}\right)\right)^{N_{2}}} \frac{\left|E_{k}\right|^{\frac{1}{p_{2}}}}{\left(2^{j} r\right)^{n-2 \beta_{1}}}\left|E_{j}\right|^{1-\frac{1}{p_{1}}}\left(2^{j} r\right)^{-2 \beta_{2}}\left\|f_{j}\right\|_{L^{p_{1}}\left(\mathbb{R}^{n}\right)}\right)^{q} \\
& \lesssim\left(1+r m_{V}\left(x_{0}\right)\right)^{\alpha} r^{-\lambda n} \sum_{k=-\infty}^{0}\left|E_{k}\right|^{\lambda}\left(\sum_{j=k+2}^{\infty} 2^{(k-j) n\left(\theta-\frac{\lambda}{q}+\frac{1}{p_{2}}\right)}\right)^{q}\|f\|_{L_{\alpha, \theta, V}^{p_{1}, q, \lambda}}^{q} \\
& \lesssim\|f\|_{L_{\alpha, \theta, V}^{p_{1}, q, \lambda}}^{q}
\end{aligned}
$$

Let $N$ be large enough. We finally get $\left\|(-\Delta+V)^{-\beta_{1}} V^{\beta_{2}} f\right\|_{L_{\alpha, \theta, V}^{p_{2}, q, \lambda}} \lesssim\|f\|_{L_{\alpha, \theta, V}^{p_{1}, q, \lambda}}$.

\section{Boundedness of the commutators on $L_{\alpha, \theta, V}^{p, q, \lambda}\left(\mathbb{R}^{n}\right)$}

In this section, let $b \in B M O\left(\mathbb{R}^{n}\right)$. We consider the boundedness of commutators $[b,(-\Delta+$ $\left.V)^{-\beta_{1}} V^{\beta_{2}}\right]$ and its duality on the generalized Morrey spaces $L_{\alpha, \theta, V}^{p, q, \lambda}\left(\mathbb{R}^{n}\right)$. For this purpose, we prove the commutator $\left[b,(-\Delta+V)^{-\beta_{1}} V^{\beta_{2}}\right]$ is bounded from $L^{p_{1}}\left(\mathbb{R}^{n}\right)$ to $L^{p_{2}}\left(\mathbb{R}^{n}\right)$. For the sake of simplicity, we denote by $b_{2^{k}}$ the mean value of $b$ on the ball $B\left(x_{0}, 2^{k} r\right)$.

Theorem 5.1 Suppose that $V \in B_{s}, s \geq \frac{n}{2}$ and $b \in B M O\left(\mathbb{R}^{n}\right)$.

(i) If $0<\beta_{2} \leq \beta_{1}<\frac{n}{2}, \frac{s}{s-\beta_{2}}<p_{1}<\frac{n}{2 \beta_{1}-2 \beta_{2}}, \frac{1}{p_{2}}=\frac{1}{p_{1}}-\frac{2 \beta_{1}-2 \beta_{2}}{n}$, then

$$
\left\|\left[b,(-\Delta+V)^{-\beta_{1}} V^{\beta_{2}}\right] f\right\|_{L^{p_{2}\left(\mathbb{R}^{n}\right)}} \lesssim\|f\|_{L^{p_{1}\left(\mathbb{R}^{n}\right)}}\|b\|_{B M O} .
$$


(ii) If $1<p_{2}<\frac{s}{\beta_{2}}$ and $\frac{1}{p_{2}}=\frac{1}{p_{1}}-\frac{2 \beta_{1}-2 \beta_{2}}{n}$, then

$$
\left\|\left[b, V^{\beta_{2}}(-\Delta+V)^{-\beta_{1}}\right] f\right\|_{L^{p_{2}\left(\mathbb{R}^{n}\right)}} \lesssim\|f\|_{L^{p_{1}\left(\mathbb{R}^{n}\right)}}\|b\|_{B M O}
$$

Proof We only prove (i). (ii) can be obtained by duality. Because $\beta_{2} \leq \beta_{1}$, we can decompose the operator $(-\Delta+V)^{-\beta_{1}} V^{\beta_{2}}$ as

$$
(-\Delta+V)^{-\beta_{1}} V^{\beta_{2}}=(-\Delta+V)^{\beta_{2}-\beta_{1}}(-\Delta+V)^{-\beta_{2}} V^{\beta_{2}} .
$$

Denote by $L^{\beta_{2}-\beta_{1}}$ and $T_{\beta_{2}}$ the operators $(-\Delta+V)^{\beta_{2}-\beta_{1}}$ and $(-\Delta+V)^{-\beta_{2}} V^{\beta_{2}}$, respectively. Then we can get

$$
\begin{aligned}
{\left[b,(-\Delta+V)^{-\beta_{1}} V^{\beta_{2}}\right] f(x) } \\
=\left[b,(-\Delta+V)^{\beta_{2}-\beta_{1}}(-\Delta+V)^{-\beta_{2}} V^{\beta_{2}}\right] f(x) \\
=b L^{\beta_{2}-\beta_{1}} T_{\beta_{2}} f(x)-L^{\beta_{2}-\beta_{1}} T_{\beta_{2}}(b f)(x) \\
=b L^{\beta_{2}-\beta_{1}} T_{\beta_{2}} f(x)-L^{\beta_{2}-\beta_{1}}\left(b T_{\beta_{2}} f(x)\right) \\
\quad+L^{\beta_{2}-\beta_{1}}\left(b T_{\beta_{2}} f(x)\right)-L^{\beta_{2}-\beta_{1}} T_{\beta_{2}}(b f)(x) \\
=\left[b, L^{\beta_{2}-\beta_{1}}\right] T_{\beta_{2}} f(x)+L^{\beta_{2}-\beta_{1}}\left[b, T_{\beta_{2}}\right] f(x) .
\end{aligned}
$$

By (1) of Corollary 4.6, we can get

$$
\begin{aligned}
\left\|\left[b,(-\Delta+V)^{-\beta_{1}} V^{\beta_{2}}\right] f\right\|_{L^{p_{2}\left(\mathbb{R}^{n}\right)}} \\
\quad \lesssim\left\|\left[b, L^{\beta_{2}-\beta_{1}}\right] T_{\beta_{2}} f\right\|_{L^{p_{2}\left(\mathbb{R}^{n}\right)}}+\left\|L^{\beta_{2}-\beta_{1}}\left[b, T_{\beta_{2}}\right] f\right\|_{L^{p_{2}\left(\mathbb{R}^{n}\right)}} \\
\quad \lesssim\left\|T_{\beta_{2}} f\right\|_{L^{p_{1}\left(\mathbb{R}^{n}\right)}}+\left\|\left[b, T_{\beta_{2}}\right] f\right\|_{L^{p_{1}\left(\mathbb{R}^{n}\right)}} \\
\quad \lesssim\|f\|_{L^{p_{1}\left(\mathbb{R}^{n}\right)} .}
\end{aligned}
$$

This completes the proof.

In the rest of this section, we prove the boundedness of the commutators $\left[b, V^{\beta_{2}}(-\Delta+\right.$ $\left.V)^{-\beta_{1}}\right]$ and $\left[b,(-\Delta+V)^{-\beta_{1}} V^{\beta_{2}}\right]$ on $L_{\alpha, \theta, V}^{p_{2}, q, \lambda}\left(\mathbb{R}^{n}\right)$, respectively.

Theorem 5.2 Suppose that $V \in B_{s}, s \geq \frac{n}{2}, \alpha \in(-\infty, 0]$ and $\lambda \in(0, n)$. Let $1<q<\infty, 1<$ $\beta_{2} \leq \beta_{1}<\frac{n}{2}$ and $1<p_{2}<\frac{s}{\beta_{2}}$ with $\frac{1}{p_{1}}-\frac{1}{p_{2}}=\frac{2 \beta_{1}-2 \beta_{2}}{n}$. If $\frac{\lambda}{q}-\frac{1}{p_{1}}+\frac{2 \beta_{1}}{n}<\theta<\frac{\lambda}{q}+1-\frac{1}{p_{1}}$, then for $b \in B M O\left(\mathbb{R}^{n}\right)$,

$$
\left\|\left[b, V^{\beta_{2}}(-\Delta+V)^{-\beta_{1}}\right] f\right\|_{L_{\alpha, \theta, V}^{p_{2}, q, \lambda}} \lesssim\|f\|_{L_{\alpha, \theta, V}^{p_{1}, q, \lambda}}\|b\|_{B M O}
$$

Proof For any ball $B\left(x_{0}, r\right)$, we have

$$
f(y)=\sum_{j=-\infty}^{\infty} f(y) \chi_{E_{j}}(y)=\sum_{j=-\infty}^{\infty} f_{j}(y)
$$


where $E_{j}=B\left(x_{0}, 2^{j} r\right) \backslash B\left(x_{0}, 2^{j-1} r\right)$. Hence, we have

$$
\begin{aligned}
\left(1+r m_{V}\left(x_{0}\right)\right)^{\alpha} r^{-\lambda n} \sum_{k=-\infty}^{0}\left|E_{k}\right|^{\theta q}\left\|\chi_{k}\left[b, V^{\beta_{2}}(-\Delta+V)^{-\beta_{1}}\right] f\right\|_{L^{p_{2}\left(\mathbb{R}^{n}\right)}}^{q} \\
\lesssim\left(1+r m_{V}\left(x_{0}\right)\right)^{\alpha} r^{-\lambda n} \sum_{k=-\infty}^{0}\left|E_{k}\right|^{\theta q}\left(\sum_{j=-\infty}^{k-2}\left\|\chi_{k}\left[b, V^{\beta_{2}}(-\Delta+V)^{-\beta_{1}}\right] f_{j}\right\|_{\left.L^{p_{2}\left(\mathbb{R}^{n}\right)}\right)}\right)^{q} \\
\quad+\left(1+r m_{V}\left(x_{0}\right)\right)^{\alpha} r^{-\lambda n} \sum_{k=-\infty}^{0}\left|E_{k}\right|^{\theta q}\left(\sum_{j=k-1}^{k+1}\left\|\chi_{k}\left[b, V^{\beta_{2}}(-\Delta+V)^{-\beta_{1}}\right] f_{j}\right\|_{\left.L^{p_{2}\left(\mathbb{R}^{n}\right)}\right)^{q}}\right. \\
\quad+\left(1+r m_{V}\left(x_{0}\right)\right)^{\alpha} r^{-\lambda n} \sum_{k=-\infty}^{0}\left|E_{k}\right|^{\theta q}\left(\sum_{j=k+2}^{\infty}\left\|\chi_{k}\left[b, V^{\beta_{2}}(-\Delta+V)^{-\beta_{1}}\right] f_{j}\right\|_{\left.L^{p_{2}\left(\mathbb{R}^{n}\right)}\right)^{q}}\right. \\
=: D_{1}+D_{2}+D_{3} .
\end{aligned}
$$

For $D_{2}$, by (ii) of Theorem 5.1, we have

$$
\begin{aligned}
D_{2} & \lesssim\left(1+r m_{V}\left(x_{0}\right)\right)^{\alpha} r^{-\lambda n} \sum_{k=-\infty}^{0}\left|E_{k}\right|^{\theta q}\left(\sum_{j=k-1}^{k+1}\left\|f_{j}\right\|_{L^{p_{1}\left(\mathbb{R}^{n}\right)}}\right)^{q}\|b\|_{B M O}^{q} \\
& \lesssim\|f\|_{L_{\alpha, \theta, V}^{p_{1}, q, \lambda}}^{q}\|b\|_{B M O}^{q} .
\end{aligned}
$$

For $D_{1}$, by Lemmas 2.7 and 4.1, we obtain

$$
\begin{aligned}
\left\|\chi_{k}\left[b, V^{\beta_{2}}(-\Delta+V)^{-\beta_{1}}\right] f_{j}\right\|_{L^{p_{2}}\left(\mathbb{R}^{n}\right)} & \vdots \frac{1}{\left(1+2^{k} r m_{V}\left(x_{0}\right)\right)^{N / k_{0}+1}} \frac{1}{\left(2^{k} r\right)^{n-2 \beta_{1}}}\left(\int_{E_{k}}\left|\int_{E_{j}} V^{\beta_{2}}(x)(b(x)-b(y)) f(y) d y\right|^{p_{2}} d x\right)^{\frac{1}{p_{2}}} \\
\lesssim & \frac{1}{\left(1+2^{k} r m_{V}\left(x_{0}\right)\right)^{N / k_{0}+1}} \frac{1}{\left(2^{k} r\right)^{n-2 \beta_{1}}}\left[\left(\int_{E_{k}} V^{\beta_{2} p_{2}}(x)\left|b(x)-b_{2^{k}}\right|^{p_{2}} d x\right)^{\frac{1}{p_{2}}} \int_{E_{j}}|f(y)| d y\right. \\
& \left.+\left(\int_{E_{k}} V^{\beta_{2} p_{2}}(x) d x\right)^{\frac{1}{p_{2}}} \int_{E_{j}}\left|b(y)-b_{2^{k} r}\right| f(y) \mid d y\right] \\
\lesssim & \frac{\|b\|_{B M O}}{\left(1+2^{k} r m_{V}\left(x_{0}\right)\right)^{N / k_{0}+1}} \frac{1}{\left(2^{k} r\right)^{n-2 \beta_{1}}}\left[\left(\int_{E_{k}} V(x) d x\right)^{\beta_{2}}\left|E_{k}\right|^{\frac{1}{p_{2}}-\beta_{2}} \int_{E_{j}}|f(y)| d y\right. \\
& \left.+\left(\int_{E_{k}} V(x) d x\right)^{\beta_{2}}\left|E_{k}\right|^{\frac{1}{p_{2}}-\beta_{2}}\left|E_{j}\right|^{1-\frac{1}{p_{1}}}(k-j)\left\|f_{j}\right\|_{L^{p_{1}}\left(\mathbb{R}^{n)}\right]}\right] \\
\lesssim & \frac{\|b\|_{B M O}}{\left(1+2^{k} r m_{V}\left(x_{0}\right)\right)^{N_{1}}} \frac{k-j}{\left(2^{k} r\right)^{n-2 \beta_{1}}}\left|E_{k}\right|^{\frac{1}{p_{2}}-\frac{2 \beta_{2}}{n}}\left|E_{j}\right|^{1-\frac{1}{p_{1}}}\left\|f_{j}\right\|_{L^{p_{1}}\left(\mathbb{R}^{n}\right)}
\end{aligned}
$$

where $\frac{1}{p_{1}}-\frac{1}{p_{2}}=\frac{2 \beta_{1}-2 \beta_{2}}{n}$ and $N_{1}<\left(N / k_{0}+1\right)-\left(\log _{2} C_{0}+1\right) \beta_{2}$. Since $\frac{\lambda}{q}-\frac{1}{p_{1}}+\frac{2 \beta_{1}}{n}<\theta<$ $\frac{\lambda}{q}+1-\frac{1}{p_{1}}$, we obtain

$$
\begin{aligned}
D_{1} \lesssim & \|b\|_{B M O}^{q}\left(1+r m_{V}\left(x_{0}\right)\right)^{\alpha} r^{-\lambda n} \sum_{k=-\infty}^{0}\left|E_{k}\right|^{\theta q} \\
& \times\left(\sum_{j=-\infty}^{k-2} \frac{1}{\left(1+2^{k} r m_{V}\left(x_{0}\right)\right)^{N_{1}}} \frac{k-j}{\left(2^{k} r\right)^{n-2 \beta_{1}}}\left|E_{k}\right|^{\frac{1}{p_{2}}-\frac{2 \beta_{2}}{n}}\left|E_{j}\right|^{1-\frac{1}{p_{1}}}\left\|f_{j}\right\|_{L^{p_{1}}\left(\mathbb{R}^{n}\right)}\right)^{q}
\end{aligned}
$$




$$
\begin{aligned}
& \lesssim\|b\|_{B M O}^{q}\left(1+r m_{V}\left(x_{0}\right)\right)^{\alpha} r^{-\lambda n} \sum_{k=-\infty}^{0}\left|E_{k}\right|^{\theta q} \\
& \quad \times\left(\sum_{j=-\infty}^{k-2} \frac{\left(1+2^{j} r m_{V}\left(x_{0}\right)\right)^{-\frac{\alpha}{q}}\left(2^{j} r\right)^{\frac{\lambda n}{q}}\left|E_{j}\right|^{-\theta}}{\left(1+2^{k} m_{V}\left(x_{0}\right)\right)^{N_{1}}} \frac{\left|E_{k}\right|^{\frac{1}{p_{2}}-\frac{2 \beta_{2}}{n}}}{\left(2^{k} r\right)^{n-2 \beta_{1}}} \frac{\left.E_{j}\right|^{\frac{1}{p_{1}}-1}}{(k-j)}\right)^{q}\|f\|_{L_{\alpha, \theta, V}^{p_{1}, q, \lambda}}^{q} \\
& \lesssim\|b\|_{B M O}^{q} \frac{\left(1+r m_{V}\left(x_{0}\right)\right)^{\alpha}}{r^{\lambda n}} \sum_{k=-\infty}^{0}\left|E_{k}\right|^{\lambda}\left(\sum_{j=-\infty}^{k-2}(k-j) 2^{(j-k) n\left(\frac{\lambda}{q}-\theta-\frac{1}{p_{1}}+1\right)}\right)^{q}\|f\|_{L_{\alpha, \theta, V}^{p_{1}, q, \lambda}}^{q} \\
& \lesssim\|f\|_{L_{\alpha, \theta, V}^{p_{1}, q, \lambda}}^{q}\|b\|_{B M O}^{q} .
\end{aligned}
$$

For $D_{3}$, because $\frac{1}{p_{1}}-\frac{1}{p_{2}}=\frac{2 \beta_{1}-2 \beta_{2}}{n}$ and $N_{1}<\left(N / k_{0}+1\right)-\left(\log _{2} C_{0}+1\right) \beta_{2}$, we have

$$
\begin{aligned}
& \left\|\chi_{k}\left[b, V^{\beta_{2}}(-\Delta+V)^{-\beta_{1}}\right] f_{j}\right\|_{L^{p_{2}\left(\mathbb{R}^{n}\right)}} \\
& \quad \lesssim \frac{1}{\left(1+2 j r m_{V}\left(x_{0}\right)\right)^{N / k_{0}+1}} \frac{1}{(2 j r)^{n-2 \beta_{1}}}\left(\int_{E_{k}}\left|\int_{E_{j}} V(x)^{\beta_{2}}(b(x)-b(y)) f(y) d y\right|^{p_{2}} d x\right)^{\frac{1}{p_{2}}} \\
& \quad \lesssim \frac{j-k}{\left(1+2 j r m_{V}\left(x_{0}\right)\right)^{N_{1}}}\left|E_{j}\right|^{\frac{2 \beta_{1}}{n}-\frac{1}{p_{1}}}\left|E_{k}\right|^{\frac{1}{p_{2}} \frac{2 \beta_{2}}{n}}\|b\|_{B M O}\left\|f_{j}\right\|_{L^{p_{1}\left(\mathbb{R}^{n}\right)},}
\end{aligned}
$$

where we have used the fact that $|x-y| \sim 2^{j} r$ for $x \in E_{k}, y \in E_{j}$ and $j \geq k+2$. Since $\frac{\lambda}{q}-\frac{1}{p_{1}}+$ $\frac{2 \beta_{1}}{n}<\theta<\frac{\lambda}{q}+1-\frac{1}{p_{1}}$, we obtain

$$
\begin{aligned}
D_{3} \lesssim & \left(1+r m_{V}\left(x_{0}\right)\right)^{\alpha} r^{-\lambda n} \sum_{k=-\infty}^{0}\left|E_{k}\right|^{\theta q} \\
& \times\left(\sum_{j=k+2}^{\infty} \frac{j-k}{\left(1+2 j r m_{V}\left(x_{0}\right)\right)^{N_{1}}}\left|E_{j}\right|^{\frac{2 \beta_{1}}{n}-\frac{1}{p_{1}}}\left|E_{k}\right|^{\frac{1}{p_{2}}-\frac{2 \beta_{2}}{n}}\|b\|_{B M O}\left\|f_{j}\right\|_{\left.L^{p_{1}\left(\mathbb{R}^{n}\right)}\right)}\right)^{q} \\
\lesssim & \|b\|_{B M O}^{q}\left(1+r m_{V}\left(x_{0}\right)\right)^{\alpha} r^{-\lambda n} \sum_{k=-\infty}^{0}\left|E_{k}\right|^{\theta q} \\
& \times\left(\sum_{j=k+2}^{\infty} \frac{\left(1+2^{j} r m_{V}\left(x_{0}\right)\right)^{-\frac{\alpha}{q}}\left(2^{j} r\right)^{\frac{\lambda n}{q}}\left|E_{j}\right|^{-\theta}}{\left(1+2^{j} r m_{V}\left(x_{0}\right)\right)^{N_{1}}} \frac{\left|E_{k}\right|^{\frac{1}{p_{2}}-\frac{2 \beta_{2}}{n}}}{\left|E_{j}\right|^{\frac{2 \beta_{1}}{n}-\frac{1}{p_{1}}}}(j-k)\right)^{q}\|f\|_{L_{\alpha, \theta, V}^{p_{1}, q, \lambda}}^{q} \\
\lesssim & \|b\|_{B M O}^{q}\left(1+r m_{V}\left(x_{0}\right)\right)^{\alpha} r^{-\lambda n} \sum_{k=-\infty}^{0}\left|E_{k}\right|^{\lambda}\left(\sum_{j=k+2}^{\infty}(j-k) 2^{(k-j) n\left(\theta-\frac{\lambda}{q}+\frac{1}{p_{1}}+\frac{2 \beta_{1}}{n}\right)}\right)^{q}\|f\|_{L_{\alpha, \theta, V}^{p_{1}, q, \lambda}}^{q} \\
\lesssim & \|f\|_{L_{\alpha, \theta, V}^{p_{1}, q, \lambda}}^{q}\|b\|_{B M O}^{q} .
\end{aligned}
$$

Let $N$ be large enough. Finally, we get

$$
\left\|\left[b, V^{\beta_{2}}(-\Delta+V)^{-\beta_{1}}\right] f\right\|_{L_{\alpha, \theta, V}^{p_{2}, q, \lambda}} \lesssim\|f\|_{L_{\alpha, \theta, V}^{p_{1}, q, \lambda}}\|b\|_{B M O}
$$

Theorem 5.3 Suppose that $V \in B_{s}, s \geq \frac{n}{2}$ and $b \in B M O\left(\mathbb{R}^{n}\right)$. Let $\alpha \in(-\infty, 0], \lambda \in(0, n)$ and $1<q<\infty$. If $0<\beta_{2} \leq \beta_{1}<\frac{n}{2}, \frac{s}{s-\beta_{2}}<p_{1}<\frac{n}{2 \beta_{1}-2 \beta_{2}}, \frac{1}{p_{2}}=\frac{1}{p_{1}}-\frac{2 \beta_{1}-2 \beta_{2}}{n}, \frac{\lambda}{q}-\frac{1}{p_{2}}<\theta<\frac{\lambda}{q}-\frac{1}{p_{2}}+$ $1-\frac{2 \beta_{1}}{n}$, then

$$
\left\|\left[b,(-\Delta+V)^{-\beta_{1}} V^{\beta_{2}}\right] f\right\|_{L_{\alpha, \theta, V}^{p_{2}, q, \lambda}} \lesssim\|f\|_{L_{\alpha, \theta, V}^{p_{1}, q, \lambda}}\|b\|_{B M O} .
$$


Proof Similarly, we can decompose $f$ based on an arbitrary ball $B\left(x_{0}, r\right)$ as follows:

$$
f(y)=\sum_{j=-\infty}^{\infty} f(y) \chi_{E_{j}}(y)=\sum_{j=-\infty}^{\infty} f_{j}(y),
$$

where $E_{j}=B\left(x_{0}, 2^{j} r\right) \backslash B\left(x_{0}, 2^{j-1} r\right)$. Hence, we have

$$
\begin{aligned}
& \left(1+r m_{V}\left(x_{0}\right)\right)^{\alpha} r^{-\lambda n} \sum_{k=-\infty}^{0}\left|E_{k}\right|^{\theta q}\left\|\chi_{k}\left[b,(-\Delta+V)^{-\beta_{1}} V^{\beta_{2}}\right] f\right\|_{L^{p_{2}\left(\mathbb{R}^{n}\right)}}^{q} \\
& \lesssim\left(1+r m_{V}\left(x_{0}\right)\right)^{\alpha} r^{-\lambda n} \sum_{k=-\infty}^{0}\left|E_{k}\right|^{\theta q}\left(\sum_{j=-\infty}^{k-2}\left\|\chi_{k}\left[b,(-\Delta+V)^{-\beta_{1}} V^{\beta_{2}}\right] f_{j}\right\|_{L^{p_{2}\left(\mathbb{R}^{n}\right)}}\right)^{q} \\
& +\left(1+r m_{V}\left(x_{0}\right)\right)^{\alpha} r^{-\lambda n} \sum_{k=-\infty}^{0}\left|E_{k}\right|^{\theta q}\left(\sum_{j=k-1}^{k+1}\left\|\chi_{k}\left[b,(-\Delta+V)^{-\beta_{1}} V^{\beta_{2}}\right] f_{j}\right\|_{L^{p_{2}\left(\mathbb{R}^{n}\right)}}\right)^{q} \\
& +\left(1+r m_{V}\left(x_{0}\right)\right)^{\alpha} r^{-\lambda n} \sum_{k=-\infty}^{0}\left|E_{k}\right|^{\theta q}\left(\sum_{j=k+2}^{\infty}\left\|\chi_{k}\left[b,(-\Delta+V)^{-\beta_{1}} V^{\beta_{2}}\right] f_{j}\right\|_{L^{p_{2}\left(\mathbb{R}^{n}\right)}}\right)^{q} \\
& =F_{1}+F_{2}+F_{3} .
\end{aligned}
$$

Applying Theorem 5.1, we can get

$$
\begin{aligned}
F_{2} & \lesssim \frac{\left(1+r m_{V}\left(x_{0}\right)\right)^{\alpha}}{r^{\lambda n}} \sum_{k=-\infty}^{0}\left|E_{k}\right|^{\theta q}\left(\sum_{j=k-1}^{k+1}\left\|f_{j}\right\|_{L^{p_{1}\left(\mathbb{R}^{n}\right)}}\right)^{q}\|b\|_{B M O}^{q} \\
& \lesssim\|f\|_{L_{\alpha, \theta, V}^{p_{1}, q, \lambda}}^{q}\|b\|_{B M O}^{q} .
\end{aligned}
$$

For $F_{1}$, by Hölder's inequality and the fact that $V \in B_{s}$, we apply Lemmas 4.1 and 2.7 to deduce that

$$
\begin{aligned}
& \left\|\chi_{k}\left[b,(-\Delta+V)^{-\beta_{1}} V^{\beta_{2}}\right] f_{j}\right\|_{L^{p_{2}\left(\mathbb{R}^{n}\right)}} \\
& \lesssim \frac{1}{\left(1+2^{k} r m_{V}\left(x_{0}\right)\right)^{N / k_{0}+1}} \frac{1}{\left(2^{k} r\right)^{n-2 \beta_{1}}}\left(\int_{E_{k}}\left|\int_{E_{j}}(b(x)-b(y)) V^{\beta_{2}}(y) f(y) d y\right|^{p_{2}} d x\right)^{\frac{1}{p_{2}}} \\
& \lesssim \frac{1}{\left(1+2^{k} r m_{V}\left(x_{0}\right)\right)^{N / k_{0}+1}} \frac{1}{\left(2^{k} r\right)^{n-2 \beta_{1}}} \\
& \times\left[\left(\int_{E_{k}}\left|b(x)-b_{2^{k}}\right|^{p_{2}} d x\right)^{\frac{1}{p_{2}}} \int_{E_{j}}\left|V^{\beta_{2}}(y) f(y)\right| d y\right. \\
& \left.+\left|E_{k}\right|^{\frac{1}{p_{2}}} \int_{E_{j}}\left|b(y)-b_{2^{k}}\right|\left|V^{\beta_{2}}(y) f(y)\right| d y\right] \\
& \lesssim \frac{\left(\int_{E_{j}} V(y) d y\right)^{\beta_{2}}}{\left(1+2^{k} r m_{V}\left(x_{0}\right)\right)^{N / k_{0}+1}} \frac{k-j}{\left(2^{k} r\right)^{n-2 \beta_{1}}}\left|E_{k}\right|^{\frac{1}{p_{2}}}\left|E_{j}\right|^{1-\frac{1}{p_{1}}}\|b\|_{B M O}\left\|f_{j}\right\|_{L^{p_{1}}\left(\mathbb{R}^{n}\right)} \\
& \lesssim\|b\|_{B M O} \frac{k-j}{\left(1+2^{k} r m_{V}\left(x_{0}\right)\right)^{N_{2}}}\left|E_{k}\right|^{\frac{1}{p_{2}}+\frac{2 \beta_{1}}{n}-1}\left|E_{j}\right|^{1-\frac{1}{p_{1}}-\frac{2 \beta_{2}}{n}}\left\|f_{j}\right\|_{L^{p_{1}\left(\mathbb{R}^{n}\right)}},
\end{aligned}
$$


where $\frac{1}{p_{2}}=\frac{1}{p_{1}}-\frac{2 \beta_{1}-2 \beta_{2}}{n}$ and $N_{2}<\left(N / k_{0}+1\right)-\left(\log _{2} C_{0}+1\right) \beta_{2}$. Since $\frac{\lambda}{q}-\frac{1}{p_{2}}<\theta<\frac{\lambda}{q}-\frac{1}{p_{2}}+$ $1-\frac{2 \beta_{1}}{n}$, we obtain

$$
\begin{aligned}
F_{1} \lesssim & \|b\|_{B M O}^{q}\left(1+r m_{V}\left(x_{0}\right)\right)^{\alpha} r^{-\lambda n} \sum_{k=-\infty}^{0}\left|E_{k}\right|^{\theta q} \\
& \times\left(\sum_{j=-\infty}^{k-2} \frac{k-j}{\left(1+2^{k} r m_{V}\left(x_{0}\right)\right)^{N_{2}}}\left|E_{k}\right|^{\frac{1}{p_{2}}+\frac{2 \beta_{1}}{n}-1}\left|E_{j}\right|^{1-\frac{1}{p_{1}}-\frac{2 \beta_{2}}{n}}\left\|f_{j}\right\|_{L^{p_{1}}\left(\mathbb{R}^{n}\right)}\right)^{q} \\
\lesssim & \|b\|_{B M O}^{q}\left(1+r m_{V}\left(x_{0}\right)\right)^{\alpha} r^{-\lambda n} \sum_{k=-\infty}^{0}\left|E_{k}\right|^{\theta q} \\
& \times\left(\sum_{j=-\infty}^{k-2} \frac{\left(1+2^{j} r m_{V}\left(x_{0}\right)\right)^{-\frac{\alpha}{q}}}{\left(1+2^{k} r m_{V}\left(x_{0}\right)\right)^{N_{2}}}\left(2^{j} r\right)^{\frac{\lambda n}{q}}\left|E_{j}\right|^{-\theta} \frac{\left|E_{j}\right|^{1-\frac{1}{p_{2}}-\frac{2 \beta_{1}}{n}}}{\left|E_{k}\right|^{1-\frac{1}{p_{2}}-\frac{2 \beta_{1}}{n}}}(k-j)\right)^{q}\|f\|_{L_{\alpha, \theta, V}^{p_{1}, q, \lambda}}^{q} \\
\lesssim & \|b\|_{B M O}^{q} \frac{\left(1+r m_{V}\left(x_{0}\right)\right)^{\alpha}}{r^{\lambda n}} \sum_{k=-\infty}^{0}\left|E_{k}\right|^{\lambda}\left(\sum_{j=-\infty}^{k-2}(k-j) 2^{(k-j) n\left(\theta-\frac{\lambda}{q}+\frac{1}{p_{2}}-1+\frac{2 \beta_{1}}{n}\right)}\right)^{q}\|f\|_{L_{\alpha, \theta, V}^{p_{1}, q, \lambda}}^{q} \\
\lesssim & \|f\|_{L_{\alpha, \theta, V}^{p_{1}, q, \lambda}}^{q}\|b\|_{B M O}^{q} .
\end{aligned}
$$

For $F_{3}$, note that when $x \in E_{k}, y \in E_{j}$ and $j \geq k+2$, then $|x-y| \sim 2^{j} r$. Similar to $F_{1}$, we have

$$
\begin{aligned}
& \left\|\chi_{k}\left[b,(-\Delta+V)^{-\beta_{1}} V^{\beta_{2}}\right] f_{j}\right\|_{L^{p_{2}\left(\mathbb{R}^{n}\right)}} \\
& \quad \lesssim \frac{1}{\left(1+2 j r m_{V}\left(x_{0}\right)\right)^{N / k_{0}+1}} \frac{1}{(2 j r)^{n-2 \beta_{1}}}\left(\int_{E_{k}}\left|\int_{E_{j}}(b(x)-b(y)) V(y)^{\beta_{2}} f(y) d y\right|^{p_{2}} d x\right)^{\frac{1}{p_{2}}} \\
& \quad \lesssim \frac{j-k}{\left(1+2 j r m_{V}\left(x_{0}\right)\right)^{N_{2}}}\left|E_{k}\right|^{\frac{1}{p_{2}}}\left|E_{j}\right|^{-\frac{1}{p_{2}}}\left\|f_{j}\right\|_{L^{p_{1}}\left(\mathbb{R}^{n}\right)}\|b\|_{B M O},
\end{aligned}
$$

where $\frac{1}{p_{2}}=\frac{1}{p_{1}}-\frac{2 \beta_{1}-2 \beta_{2}}{n}$ and $N_{2}<\left(N / k_{0}+1\right)-\left(\log _{2} C_{0}+1\right) \beta_{2}$. Since $\frac{\lambda}{q}-\frac{1}{p_{2}}<\theta<\frac{\lambda}{q}-\frac{1}{p_{2}}+$ $1-\frac{2 \beta_{1}}{n}$, we obtain

$$
\begin{aligned}
& F_{3} \lesssim\|b\|_{B M O}^{q}\left(1+r m_{V}\left(x_{0}\right)\right)^{\alpha} r^{-\lambda n} \sum_{k=-\infty}^{0}\left|E_{k}\right|^{\theta q} \\
& \times\left(\sum_{j=k+2}^{\infty} \frac{\left(1+2^{j} r m_{V}\left(x_{0}\right)\right)^{-\frac{\alpha}{q}}}{\left(1+2^{j} r m_{V}\left(x_{0}\right)\right)^{N_{2}}}\left(2^{j} r\right)^{\frac{\lambda n}{q}}\left|E_{j}\right|^{-\theta} \frac{\left|E_{k}\right|^{\frac{1}{p_{2}}}}{\left|E_{j}\right|^{\frac{1}{p_{2}}}}(j-k)\left\|f_{j}\right\|_{L^{p_{1}\left(\mathbb{R}^{n}\right)}}\right)^{q} \\
& \lesssim\|b\|_{B M O}^{q}\left(1+r m_{V}\left(x_{0}\right)\right)^{\alpha} r^{-\lambda n} \sum_{k=-\infty}^{0}\left|E_{k}\right|^{\lambda}\left(\sum_{j=k+2}^{\infty}(j-k) 2^{(k-j) n\left(\theta-\frac{\lambda}{q}+\frac{1}{p_{2}}\right)}\right)^{q}\|f\|_{L_{\alpha, \theta, V}^{p_{1}, \lambda, \lambda}}^{q} \\
& \lesssim\|f\|_{L_{\alpha, \theta, V}^{p_{1}, q, \lambda}}^{q}\|b\|_{B M O}^{q}
\end{aligned}
$$

Let $N$ be large enough. We finally get

$$
\left\|\left[b,(-\Delta+V)^{-\beta_{1}} V^{\beta_{2}}\right] f\right\|_{L_{\alpha, \theta, V}^{p_{2}, q, \lambda}} \lesssim\|f\|_{L_{\alpha, \theta, V}^{p_{1}, q, \lambda}}\|b\|_{B M O}
$$


Competing interests

The authors declare that they have no competing interests.

\section{Authors' contributions}

All authors read and approved the final manuscript.

\section{Author details}

${ }^{1}$ College of Mathematics, Qingdao University, Qingdao, Shandong 266071, China. ${ }^{2}$ Department of Mathematics, Shantou University, Shantou, Guangdong 515063, China.

\section{Acknowledgements}

Project was supported by NSFC No. 11171203; New Teacher's Fund for Doctor Stations, Ministry of Education No. 20114402120003; Guangdong Natural Science Foundation S2011040004131; Foundation for Distinguished Young Talents in Higher Education of Guangdong, China, LYM11063.

Received: 25 October 2014 Accepted: 1 July 2015 Published online: 23 July 2015

\section{References}

1. Shen, Z: $L^{P}$ Estimate for Schrödinger operator with certain potentials. Ann. Inst. Fourier 45, 513-546 (1995)

2. Guo, Z, Li, P, Peng, L: L $L^{p}$ Boundedness of commutators of Riesz transforms associated to Schrödinger operator. J. Math. Anal. Appl. 341, 421-432 (2008)

3. Liu, Y: The weighted estimates for the operators $V^{\alpha}\left(-\Delta_{G}+V\right)^{-\beta}$ and $V^{\alpha} \nabla_{G}\left(-\Delta_{G}+V\right)^{-\beta}$ on the stratified Lie group $G$. J. Math. Anal. Appl. 349, 235-244 (2009)

4. Liu, Y, Huang, J, Dong, J: Commutators of Calderón-Zygmund operators related to admissible functions on spaces of homogeneous type and applications to Schrödinger operators. Sci. China Math. 56, 1895-1913 (2013)

5. Liu, Y, Wang, L, Dong, J: Commutators of higher order Riesz transform associated with Schrödinger operators. J. Funct. Spaces Appl. 2013, Article ID 842375 (2013)

6. Tang, L, Dong, J: Boundedness for some Schrödinger type operators on Morrey spaces related to certain nonnegative potentials. J. Math. Anal. Appl. 355, 101-109 (2009)

7. Yang, DC, Yang, DY, Zhou, Y: Endpoint properties of localized Riesz transforms and fractional integrals associated to Schrödinger operators. Potential Anal. 30, 271-300 (2009)

8. Yang, DC, Yang, DY, Zhou, Y: Localized BMO and BLO spaces on RD-spaces and applications to Schrödinger operators. Commun. Pure Appl. Anal. 9, 779-812 (2010)

9. Sugano, S: Estimates for the operators $V^{\alpha}(-\Delta+V)^{-\beta}$ and $V^{\alpha} \nabla(-\Delta+V)^{-\beta}$ with certain non-negative potentials $V$. Tokyo J. Math. 21, 441-452 (1998)

10. Peetre, J: On the theory of $\mathcal{L}_{p, \lambda}$ spaces. J. Funct. Anal. 4, 71-87 (1969)

11. Herz, C: Lipschitz spaces and Bernstein's theorem on absolutely convergent Fourier transforms. J. Math. Mech. 18 283-323 (1968/1969)

12. Chen, $X$, Chen, J: Boundedness of sublinear operators on generalized Morrey spaces and its application. Chin. Ann. Math., Ser. A 32, 705-720 (2011)

13. Lu, $\mathrm{S}, \mathrm{Xu}, \mathrm{L}$ : Boundedness of rough singular integral operators on the homogeneous Morrey-Herz spaces. Hokkaido Math. J. 34, 299-314 (2005)

14. Duong, X, Yan, L: On commutators of fractional integrals. Proc. Am. Math. Soc. 132, 3549-3557 (2004)

15. Jiang, Y: Endpoint estimates for fractional integral associated to Schrödinger operators on the Heisenberg groups. Acta Math. Sci., Ser. B. 31, 993-1000 (2011)

16. Coifman, R, Rochberg, R, Weiss, G: Factorization theorems for Hardy space in several variables. Ann. Math. 103 611-635 (1988)

17. Liu, Y: Commutators of $B M O$ functions and degenerate Schrödinger operators with certain nonnegative potentials. Monatshefte Math. 165, 41-56 (2012)

18. Dziubański, J, Zienkiewicz, J: HP Spaces for Schrödinger operator. In: Fourier Analysis and Related Topics. Banach Center Publ., vol. 56, pp. 45-53 (2002)

19. John, F, Nirenberg, L: On function of bounded mean oscillation. Commun. Pure Appl. Math. 14, 415-426 (1961)

20. Bui, T-A: Weighted estimates for commutators of some singular integrals related to Schrödinger operators. Bull. Sci. Math. 138, 270-292 (2014)

\section{Submit your manuscript to a SpringerOpen ${ }^{\circ}$ journal and benefit from:}

- Convenient online submission

Rigorous peer review

- Immediate publication on acceptance

- Open access: articles freely available online

- High visibility within the field

- Retaining the copyright to your article 